\title{
Classification of Relativity
}

\author{
Vu B. Ho \\ Advanced Study, 9 Adela Court, Mulgrave, Victoria, Australia \\ Email:vubho@bigpond.net.au
}

How to cite this paper: Ho, V.B. (2020) Classification of Relativity. Journal of Modern Physics, 11, 535-564.

https://doi.org/10.4236/jmp.2020.114036

Received: March 20, 2020

Accepted: April 7, 2020

Published: April 10, 2020

Copyright $@ 2020$ by author(s) and Scientific Research Publishing Inc. This work is licensed under the Creative Commons Attribution International License (CC BY 4.0).

http://creativecommons.org/licenses/by/4.0/

\begin{abstract}
In this work, we discuss the possibility to classify relativity in accordance with the classification of second order partial differential equations that have been applied into the formulation of physical laws in physics. In mathematics, since second order partial differential equations can be classified into hyperbolic, elliptic or parabolic type, therefore we show that it is also possible to classify relativity accordingly into hyperbolic, elliptic or parabolic type by establishing coordinate transformations that preserve the forms of these second order partial differential equations. The coordinate transformation that preserves the form of the hyperbolic equation is the Lorentz transformation and the associated space is the hyperbolic, or pseudo-Euclidean, relativistic spacetime. Typical equations in physics that comply with hyperbolic relativity are Maxwell and Dirac equations. The coordinate transformation that preserves the form of the elliptic equation is the modified Lorentz transformation that we have formulated in our work on Euclidean relativity and the associated space is the elliptic, or Euclidean, relativistic spacetime. As we will show in this work, equations that comply with elliptic relativity are the equations that describe the subfields of Maxwell and Dirac field. And the coordinate transformation that preserves the form of the parabolic equation is the Euclidean transformation consisting of the translation and rotation in the spatial space and the associated space is the parabolic relativistic spacetime, which is a Euclidean space with a universal time. Typical equations in physics that comply with parabolic relativity are the diffusion equation, the Schrödinger equation and in particular the diffusion equations that are derived from the four-current defined in terms of the differentiable structures of the spacetime manifold, and the Ricci flow.
\end{abstract}

\section{Keywords}

Special Relativity, Elliptic Equation, Hyperbolic Equation, Parabolic Equation, Elliptic Relativity, Hyperbolic Relativity, Parabolic Relativity, Classification of Relativity, Maxwell and Dirac Field, Maxwell and Dirac Subfield 


\section{Introduction}

In physics, it appears that physical objects are endowed with many different physical properties each of which couples to a physical field that obeys a specific physical law that can be described by a particular system of partial differential equations. It is also conventionally assumed, due to our ability of observing and perceiving of the natural environment, that physical events occur in a three-dimensional space and progress forward in one-dimensional time, even though it is conceivable to speculate that physical events may also progress backwards in time and occur in a higher dimensional space. From the physical laws that are derived and formulated from observation, a mathematical structure of space and time can be constructed to conform to the corresponding observed physical occurrences. In Newton physics since the established dynamical laws that describe the dynamics of material particles seem to obey the Galilean transformation of space and time therefore it is reasonable to assume that time is absolute. On the other hand, in Einstein physics, space and time are relative since it is established that Maxwell field equations of the electromagnetic field comply with the Lorentz transformation. Maxwell field equations are wave equations that describe the dynamics of a wave motion rather than that of a material particle. Until the quantum mechanics was invented which embraces the wave-particle dual characteristics of a material particle, it had been regarded that Newton and Maxwell dynamics are two different dynamics that describe physical systems that have completely different physical compositions, even though Newton himself speculated that the electromagnetic field is also composed of particles. The difficulty associated with the wave-particle duality may be due to the assumption that an elementary particle such as an electron is simply a mass-point with no internal structure. In fact, we have shown that it is possible to describe mathematically an elementary particle as a three-dimensional differentiable manifold whose mathematical structure can be expressed in terms of a Schrödinger wavefunction. Therefore, from the superposition principle associated with the wave motion and the assumption of internal structures of an elementary particle, we may assume that a physical property endowed to an elementary particle does not have to satisfy the requirements that are imposed on other physical properties of the particle but rather follows its own physical law that obeys its own type of relativity. For example, in quantum mechanics the time-independent Schrödinger wave equation describes the structure of atoms and it has been shown that atoms are stable and their physical structures are invariant with respect to translation and rotation, and we have also shown that the spin dynamics can be formulated by the Schrödinger equation in terms of intrinsic coordinates rather than the Dirac relativistic equation, therefore the Schrödinger equation should not be considered as a non-relativistic limit of Dirac relativistic equation but rather a physical formulation that follows its own relativity and as we will show later that the relativity that the Schrödinger equation obeys is the parabolic relativity, in the same way as Maxwell and Dirac field to comply with the pseudo-Euclidean 
relativity.

In this work, we discuss a classification of relativity in which the spacetime manifold in which physical phenomena occur are classified into hyperbolic, elliptic or parabolic relativistic spacetime. Since the classification of relativity is closely related to the classification of second order partial differential equations therefore for reference we first outline the classification of the second order partial differential equations in Section 2, and the classification of relativity will be given in Section 4. A hyperbolic relativistic spacetime is a pseudo-Euclidean space that was formulated by Minkowski to establish a mathematical foundation for Einstein's theory of special relativity. That is a four-dimensional differentiable manifold which possesses a fundamental quadratic form of Lorentz signature that makes the wave equation invariant under Lorentz transformation [1]. On the other hand, we have shown in our work on the Euclidean relativity that quantum particles may possess physical properties that comply with the Euclidean relativity rather than the pseudo-Euclidean relativity. Since this type of relativity is associated with the elliptic equation therefore we will refer to the spacetime continuum whose mathematical structure complies with the Euclidean relativity an elliptic relativistic spacetime. And we have also shown in our work on Euclidean relativity that the elliptic equations are invariant under a modified Lorentz transformation, which is a rotation in spacetime [2] [3]. The two types of relativistic spacetime that we have considered depend essentially on the corresponding second order partial differential equations that are used to describe possible physical properties associated with a quantum particle. In fact, in Section 3 we show that the Euclidean relativity is the spacetime structure that is associated with the subfields of the Maxwell and Dirac field, in which the dynamics of the subfields is described by elliptic equations. In addition to the elliptic and hyperbolic relativity, in Section 4 we also discuss the parabolic relativity. As it is well-known that second order partial differential equations can be classified into three distinctive types of equations therefore it seems appropriate also to classify relativistic spacetime into three different types, and the third type of relativistic spacetime that we introduce in this work is the parabolic relativistic spacetime. Therefore, by definition, a parabolic relativistic spacetime is a space whose mathematical structure is determined by the invariance of a parabolic equation such as the diffusion equation and the Schrödinger wave equation in quantum mechanics. Overall, we assume that a quantum particle may have different physical properties which are described by different physical laws each of which is formulated independently in either the hyperbolic or the elliptic or the parabolic relativistic spacetime. All of these relativistic spaces can be regarded as different fibres of the fibre bundle of the spacetime continuum.

\section{A Classification of Second Order Partial Differential Equations}

A general second order partial differential equation can be written in the form: 


$$
\sum_{i=1}^{n} \sum_{j=1}^{n} a_{i j} \frac{\partial^{2} \psi}{\partial x_{i} \partial x_{j}}+\sum_{i=1}^{n} b_{i} \frac{\partial \psi}{\partial x_{i}}+c \psi+d=0
$$

If the matrix $A=\left(a_{i j}\right)$ is symmetric then it can be transformed into a diagonal matrix by applying a diagonalising matrix $M$

$$
M^{\mathrm{T}} A M=\left(\begin{array}{ccc}
\lambda_{1} & \cdots & 0 \\
\vdots & \ddots & \vdots \\
0 & \cdots & \lambda_{n}
\end{array}\right)
$$

Then the second order partial differential equation given in Equation (1) can be classified into three different types of partial differential equations as follows [4].

- If all eigenvalues $\lambda_{i}$ are non-zero and have the same sign then Equation (1) is elliptic.

- If all eigenvalues $\lambda_{i}$ are non-zero and have the same sign except for one of the eigenvalues then Equation (1) is hyperbolic.

- If exactly one of the eigenvalues is zero and all the others have the same sign then Equation (1) is parabolic. In this case, the matrix $A$ is singular.

In this work, we consider the second order partial differential equations, and classify relativity accordingly, in the spacetime continuum in which space has three dimensions and time has one dimension therefore we only need to present the case of the partial differential equations in the four-dimensional space whose coordinates are specified by three spatial coordinates $(x, y, z)$ and one temporal coordinate $t$. With this specification, the three different types of second order partial differential equations are given as follows:

- Elliptic equation can be written in the form

$$
\frac{\partial^{2} \psi}{\partial t^{2}}+\frac{\partial^{2} \psi}{\partial x^{2}}+\frac{\partial^{2} \psi}{\partial y^{2}}+\frac{\partial^{2} \psi}{\partial z^{2}}=a \frac{\partial \psi}{\partial t}+b \frac{\partial \psi}{\partial x}+c \frac{\partial \psi}{\partial y}+d \frac{\partial \psi}{\partial z}+e \psi+f
$$

We will show in the next section that elliptic equations in the four-dimensional spacetime manifold play an important role in the determination of the dynamics of the subfields of Maxwell and Dirac field [5]. Therefore, the subfields of Maxwell and Dirac field comply with the Euclidean relativity which we will classify as elliptic relativity in this work.

- Hyperbolic equation can be written in the form

$$
\frac{\partial^{2} \psi}{\partial t^{2}}-\frac{\partial^{2} \psi}{\partial x^{2}}-\frac{\partial^{2} \psi}{\partial y^{2}}-\frac{\partial^{2} \psi}{\partial z^{2}}=a \frac{\partial \psi}{\partial t}+b \frac{\partial \psi}{\partial x}+c \frac{\partial \psi}{\partial y}+d \frac{\partial \psi}{\partial z}+e \psi+f
$$

Hyperbolic equations play an important role in physics with Maxwell theory of the electromagnetic field and Dirac theory of quantum particles [6] [7]. In particular, the invariance of the hyperbolic equations under Lorentz transformation led Einstein to develop his theories of special and general relativity. We will classify Einstein relativity as hyperbolic relativity in this work.

- Parabolic equation can be written in the form

$$
\frac{\partial^{2} \psi}{\partial x^{2}}+\frac{\partial^{2} \psi}{\partial y^{2}}+\frac{\partial^{2} \psi}{\partial z^{2}}=a \frac{\partial \psi}{\partial t}+b \frac{\partial \psi}{\partial x}+c \frac{\partial \psi}{\partial y}+d \frac{\partial \psi}{\partial z}+e \psi+f
$$


We will classify as parabolic relativity for the mathematical structure of the spacetime manifold that makes the parabolic equations invariant. The important parabolic equations in physics are the diffusion equation, the Schrödinger equation, and diffusion equations that are derived from the four-current associated with the differentiable structure of the spacetime manifold and the Ricci flow. The parabolic relativity complies with the Euclidean transformation which consists of translation and rotation in the spatial space.

\section{Subfield Structure of Maxwell and Dirac Field}

In this section, we will discuss possible physical fields that comply with the elliptic equation given in Equation (3). We have shown in our previous works that both Maxwell field equations of the electromagnetic field and Dirac equation of massive quantum particles can be formulated from a general system of linear first order partial differential equations, and, as a consequence, the field equations of the two physical fields have many common features that specify characteristics that are not typical in classical physics [8] [9] [10] [11]. In the following, we further show the similarity between the Maxwell and Dirac field by examining the subfields that are coupled to form either of these two physical fields. We show that the subfields have the mathematical structures and physical properties that are essentially different from the coupled field of Maxwell, and that of Dirac. In particular, we show that the subfields of both Maxwell and Dirac field satisfy elliptic equations rather than hyperbolic equations therefore while Maxwell and Dirac field are described by wave equations therefore they comply with the laws of the pseudo-Euclidean relativity, the Maxwell and Dirac subfields are described by elliptic equations therefore they comply with those of the Euclidean relativity instead [12]. The fact that the subfields of Maxwell and Dirac fields are Euclidean relativistic has profound implications, such as they can be used to explain the stability of elementary particles because if elementary particles are represented by subfields which are described by elliptic equations then since elliptic equations are used to describe equilibrium states of physical systems therefore elementary particles associated with those subfields are also stable. Furthermore, if quantum particles possess physical properties that are represented by subfields which are described by elliptic equations, hence acting in accordance with the Euclidean relativity, then they can be used to explain physical phenomena that require physical transmissions with speeds greater than the speed of light in vacuum, such as the Einstein-Podosky-Rosen paradox in quantum entanglement [13] [14] [15].

The system of linear first order partial differential equations that we need to use in this work is given as follows [16] [17]

$$
\sum_{i=1}^{n} \sum_{j=1}^{n} a_{i j}^{r} \frac{\partial \psi_{i}}{\partial x_{j}}=k_{1} \sum_{l=1}^{n} b_{l}^{r} \psi_{l}+k_{2} c^{r}, \quad r=1,2, \cdots, n
$$

Equation (6) can be rewritten in a matrix form as: 


$$
\left(\sum_{i=1}^{n} A_{i} \frac{\partial}{\partial x_{i}}\right) \psi=k_{1} \sigma \psi+k_{2} J
$$

where $\psi=\left(\psi_{1}, \psi_{2}, \cdots, \psi_{n}\right)^{\mathrm{T}}, \quad \partial \psi / \partial x_{i}=\left(\partial \psi_{1} / \partial x_{i}, \partial \psi_{2} / \partial x_{i}, \cdots, \partial \psi_{n} / \partial x_{i}\right)^{\mathrm{T}}, \quad A_{i}$, $\sigma$ and $J$ are matrices representing the quantities $a_{i j}^{k}, b_{l}^{r}$ and $c^{r}$, and $k_{1}$ and $k_{2}$ are undetermined constants. Now, if we apply the operator $\sum_{i=1}^{n} A_{i} \partial / \partial x_{i}$ on the left on both sides of Equation (7) then we obtain

$$
\left(\sum_{i=1}^{n} A_{i} \frac{\partial}{\partial x_{i}}\right)\left(\sum_{j=1}^{n} A_{j} \frac{\partial}{\partial x_{j}}\right) \psi=\left(\sum_{i=1}^{n} A_{i} \frac{\partial}{\partial x_{i}}\right)\left(k_{1} \sigma \psi+k_{2} J\right)
$$

If we assume further that the coefficients $a_{i j}^{k}$ and $b_{l}^{r}$ are constants and $A_{i} \sigma=\sigma A_{i}$, then Equation (8) can be rewritten in the following form

$$
\begin{aligned}
& \left(\sum_{i=1}^{n} A_{i}^{2} \frac{\partial^{2}}{\partial x_{i}^{2}}+\sum_{i=1}^{n} \sum_{j>i}^{n}\left(A_{i} A_{j}+A_{j} A_{i}\right) \frac{\partial^{2}}{\partial x_{i} \partial x_{j}}\right) \psi \\
& =k_{1}^{2} \sigma^{2} \psi+k_{1} k_{2} \sigma J+k_{2} \sum_{i=1}^{n} A_{i} \frac{\partial J}{\partial x_{i}}
\end{aligned}
$$

In order for the above systems of partial differential equations to be applied to physical phenomena, the matrices $A_{i}$ must be determined. For the case of Maxwell and Dirac field, the matrices $A_{i}$ must take a form so that Equation (9) reduces to a wave equation

$$
\left(\sum_{i=1}^{n} A_{i}^{2} \frac{\partial^{2}}{\partial x_{i}^{2}}\right) \psi=k_{1}^{2} \sigma^{2} \psi+k_{1} k_{2} \sigma J+k_{2} \sum_{i=1}^{n} A_{i} \frac{\partial J}{\partial x_{i}}
$$

From Equation (9), for Dirac field, we simply require the matrices $A_{i}$ to satisfy the conditions $A_{i} A_{j}+A_{j} A_{i}=0$ and $A_{i}^{2}= \pm 1$. However, for the case of Maxwell field, the conditions required for the matrices $A_{i}$ can be determined from the classical form of Maxwell field equations [3] [18]. Furthermore, as shown in the next subsection, in order to reduce Equation (9) to Equation (10) for the case of Maxwell field, we will also need an extra condition on the components of the wavefuction $\psi$ in the form of a divergence or Gauss's law

$$
\sum_{i=1}^{n} \frac{\partial \psi_{i}}{\partial x_{i}}=\rho
$$

In this work, we will discuss only Maxwell and Dirac field therefore we will set $\sigma=1$.

\subsection{Maxwell Field as a Coupling of Two Elliptic Fields}

In this subsection, we show that Maxwell field of electromagnetism is a coupled field that is formed from the coupling of two subfields that satisfy an elliptic equation. In order to distinguish a field that satisfies an elliptic equation from a field that satisfies a hyperbolic equation, or wave equation, we refer to the former as an elliptic field and the latter as a hyperbolic field. From the general equation given in Equation (7), the two subfields that are coupled to form the Maxwell field can be rewritten in the following simple form: 


$$
\left(A_{0} \frac{\partial}{\partial t}+A_{1} \frac{\partial}{\partial x_{1}}+A_{2} \frac{\partial}{\partial x_{2}}+A_{3} \frac{\partial}{\partial x_{3}}\right) \psi=k_{1} \psi+k_{2} J
$$

where $\psi=\left(\psi_{1}, \psi_{2}, \psi_{3}\right)^{\mathrm{T}}$ and $J=\left(j_{1}, j_{2}, j_{3}\right)^{\mathrm{T}}$, and the matrices $A_{i}$ are given as follows

$$
\begin{aligned}
& A_{0}=\mp\left(\begin{array}{ccc}
1 & 0 & 0 \\
0 & 1 & 0 \\
0 & 0 & 1
\end{array}\right), A_{1}=\left(\begin{array}{ccc}
0 & 0 & 0 \\
0 & 0 & -1 \\
0 & 1 & 0
\end{array}\right), \\
& A_{2}=\left(\begin{array}{ccc}
0 & 0 & 1 \\
0 & 0 & 0 \\
-1 & 0 & 0
\end{array}\right), A_{3}=\left(\begin{array}{ccc}
0 & -1 & 0 \\
1 & 0 & 0 \\
0 & 0 & 0
\end{array}\right)
\end{aligned}
$$

In Equation (13), the negative sign, or negative time, in front of the matrix $A_{0}$ together with other matrices form one subfield and the positive sign, or positive time, in front of the matrix $A_{0}$ together with other matrices form another subfield. Then we obtain the following results

$$
\begin{gathered}
A_{0}^{2}=\left(\begin{array}{lll}
1 & 0 & 0 \\
0 & 1 & 0 \\
0 & 0 & 1
\end{array}\right), A_{1}^{2}=\left(\begin{array}{ccc}
0 & 0 & 0 \\
0 & -1 & 0 \\
0 & 0 & -1
\end{array}\right), \\
A_{2}^{2}=\left(\begin{array}{ccc}
-1 & 0 & 0 \\
0 & 0 & 0 \\
0 & 0 & -1
\end{array}\right), A_{3}^{2}=\left(\begin{array}{ccc}
-1 & 0 & 0 \\
0 & -1 & 0 \\
0 & 0 & 0
\end{array}\right) \\
A_{0} A_{1}+A_{1} A_{0}=\mp 2 A_{i} \text { for } i=1,2,3 \\
A_{1} A_{2}+A_{2} A_{1}=\left(\begin{array}{lll}
0 & 1 & 0 \\
1 & 0 & 0 \\
0 & 0 & 0
\end{array}\right), A_{1} A_{3}+A_{3} A_{1}=\left(\begin{array}{lll}
0 & 0 & 1 \\
0 & 0 & 0 \\
1 & 0 & 0
\end{array}\right), \\
A_{2} A_{3}+A_{3} A_{2}=\left(\begin{array}{lll}
0 & 0 & 0 \\
0 & 0 & 1 \\
0 & 1 & 0
\end{array}\right)
\end{gathered}
$$

Using the matrices $A_{i}$ given in Equation (13) with the negative sign for the matrix $A_{0}$ we obtain the following system of differential equations from Equation (12)

$$
\begin{aligned}
& -\frac{\partial \psi_{1}}{\partial t}+\frac{\partial \psi_{3}}{\partial x_{2}}-\frac{\partial \psi_{2}}{\partial x_{3}}=k_{1} \psi_{1}+k_{2} j_{1} \\
& -\frac{\partial \psi_{2}}{\partial t}-\frac{\partial \psi_{3}}{\partial x_{1}}+\frac{\partial \psi_{1}}{\partial x_{3}}=k_{1} \psi_{2}+k_{2} j_{2} \\
& -\frac{\partial \psi_{3}}{\partial t}+\frac{\partial \psi_{2}}{\partial x_{1}}-\frac{\partial \psi_{1}}{\partial x_{2}}=k_{1} \psi_{3}+k_{2} j_{3}
\end{aligned}
$$

Similarly, using the matrices $A_{i}$ given in Equation (13) with the positive sign for the matrix $A_{0}$ we obtain the following system of differential equations from Equation (12) 


$$
\begin{aligned}
& \frac{\partial \psi_{4}}{\partial t}+\frac{\partial \psi_{6}}{\partial x_{2}}-\frac{\partial \psi_{5}}{\partial x_{3}}=k_{1} \psi_{4}+k_{2} j_{4} \\
& \frac{\partial \psi_{5}}{\partial t}-\frac{\partial \psi_{6}}{\partial x_{1}}+\frac{\partial \psi_{4}}{\partial x_{3}}=k_{1} \psi_{5}+k_{2} j_{5} \\
& \frac{\partial \psi_{6}}{\partial t}+\frac{\partial \psi_{5}}{\partial x_{1}}-\frac{\partial \psi_{4}}{\partial x_{2}}=k_{1} \psi_{6}+k_{2} j_{6}
\end{aligned}
$$

In Equations (20)-(22), we have used different subscripts for the field components $\psi_{i}$ because it is a different field from the field given in Equations (17)-(19). However, for simplicity, we have used the same $k_{1}$ and $k_{2}$ for the system of equations given in Equations (20)-(22) even though they may have different dimensional values from those given in Equations (17)-(19).

On the other hand, using the matrices $A_{i}$ given in Equation (13) with negative sign for the matrix $A_{0}$ we obtain the following system of differential equations from Equation (9).

$$
\begin{aligned}
& \frac{\partial^{2} \psi_{1}}{\partial t^{2}}-\frac{\partial^{2} \psi_{1}}{\partial x_{2}^{2}}-\frac{\partial^{2} \psi_{1}}{\partial x_{3}^{2}}-2 \frac{\partial}{\partial t}\left(\frac{\partial \psi_{3}}{\partial x_{2}}-\frac{\partial \psi_{2}}{\partial x_{3}}\right)+\frac{\partial}{\partial x_{1}}\left(\frac{\partial \psi_{2}}{\partial x_{2}}+\frac{\partial \psi_{3}}{\partial x_{3}}\right) \\
& =k_{1}^{2} \psi_{1}+k_{1} k_{2} j_{1}+k_{2}\left(-\frac{\partial j_{1}}{\partial t}+\frac{\partial j_{3}}{\partial x_{2}}-\frac{\partial j_{2}}{\partial x_{3}}\right) \\
& \frac{\partial^{2} \psi_{2}}{\partial t^{2}}-\frac{\partial^{2} \psi_{2}}{\partial x_{1}^{2}}-\frac{\partial^{2} \psi_{2}}{\partial x_{3}^{2}}+2 \frac{\partial}{\partial t}\left(\frac{\partial \psi_{3}}{\partial x_{1}}-\frac{\partial \psi_{1}}{\partial x_{3}}\right)+\frac{\partial}{\partial x_{2}}\left(\frac{\partial \psi_{1}}{\partial x_{1}}+\frac{\partial \psi_{3}}{\partial x_{3}}\right) \\
& =k_{1}^{2} \psi_{2}+k_{1} k_{2} j_{2}+k_{2}\left(-\frac{\partial j_{2}}{\partial t}+\frac{\partial j_{1}}{\partial x_{3}}-\frac{\partial j_{3}}{\partial x_{1}}\right) \\
& \frac{\partial^{2} \psi_{3}}{\partial t^{2}}-\frac{\partial^{2} \psi_{3}}{\partial x_{1}^{2}}-\frac{\partial^{2} \psi_{3}}{\partial x_{2}^{2}}-2 \frac{\partial}{\partial t}\left(\frac{\partial \psi_{2}}{\partial x_{1}}-\frac{\partial \psi_{1}}{\partial x_{2}}\right)+\frac{\partial}{\partial x_{3}}\left(\frac{\partial \psi_{1}}{\partial x_{1}}+\frac{\partial \psi_{2}}{\partial x_{2}}\right) \\
& =k_{1}^{2} \psi_{3}+k_{1} k_{2} j_{3}+k_{2}\left(-\frac{\partial j_{3}}{\partial t}+\frac{\partial j_{2}}{\partial x_{1}}-\frac{\partial j_{1}}{\partial x_{2}}\right)
\end{aligned}
$$

Similarly, using the matrices $A_{i}$ given in Equation (13) with positive sign for the matrix $A_{0}$ we obtain the following system of differential equations also from Equation (9).

$$
\begin{aligned}
& \frac{\partial^{2} \psi_{4}}{\partial t^{2}}-\frac{\partial^{2} \psi_{4}}{\partial x_{2}^{2}}-\frac{\partial^{2} \psi_{4}}{\partial x_{3}^{2}}+2 \frac{\partial}{\partial t}\left(\frac{\partial \psi_{6}}{\partial x_{2}}-\frac{\partial \psi_{5}}{\partial x_{3}}\right)+\frac{\partial}{\partial x_{1}}\left(\frac{\partial \psi_{5}}{\partial x_{2}}+\frac{\partial \psi_{6}}{\partial x_{3}}\right) \\
& =k_{1}^{2} \psi_{4}+k_{1} k_{2} j_{4}+k_{2}\left(\frac{\partial j_{4}}{\partial t}+\frac{\partial j_{6}}{\partial x_{2}}-\frac{\partial j_{5}}{\partial x_{3}}\right) \\
& \frac{\partial^{2} \psi_{5}}{\partial t^{2}}-\frac{\partial^{2} \psi_{5}}{\partial x_{1}^{2}}-\frac{\partial^{2} \psi_{5}}{\partial x_{3}^{2}}-2 \frac{\partial}{\partial t}\left(\frac{\partial \psi_{6}}{\partial x_{1}}-\frac{\partial \psi_{4}}{\partial x_{3}}\right)+\frac{\partial}{\partial x_{2}}\left(\frac{\partial \psi_{4}}{\partial x_{1}}+\frac{\partial \psi_{6}}{\partial x_{3}}\right) \\
& =k_{1}^{2} \psi_{5}+k_{1} k_{2} j_{5}+k_{2}\left(\frac{\partial j_{5}}{\partial t}+\frac{\partial j_{6}}{\partial x_{2}}-\frac{\partial j_{4}}{\partial x_{3}}\right) \\
& \frac{\partial^{2} \psi_{6}}{\partial t^{2}}-\frac{\partial^{2} \psi_{6}}{\partial x_{1}^{2}}-\frac{\partial^{2} \psi_{6}}{\partial x_{2}^{2}}+2 \frac{\partial}{\partial t}\left(\frac{\partial \psi_{5}}{\partial x_{1}}-\frac{\partial \psi_{4}}{\partial x_{2}}\right)+\frac{\partial}{\partial x_{3}}\left(\frac{\partial \psi_{4}}{\partial x_{1}}+\frac{\partial \psi_{5}}{\partial x_{2}}\right) \\
& =k_{1}^{2} \psi_{6}+k_{1} k_{2} j_{6}+k_{2}\left(\frac{\partial j_{6}}{\partial t}+\frac{\partial j_{5}}{\partial x_{1}}-\frac{\partial j_{4}}{\partial x_{2}}\right)
\end{aligned}
$$


The equations given in Equations (23)-(28) contain cross derivatives that involve both space and time. Even though the cross derivatives that involve the time coordinate can be removed by using the system of equations given in Equations (17)-(19) and Equations (20)-(22), the cross derivatives that involve the spatial coordinates can only be removed by imposing on the wave function $\psi$ an additional condition that is commonly known as the divergence of a vector field as given in Equation (11). The divergence of a field in fact endows the field with a physical character and gives a direct relationship between a mathematical object and a physical entity. Using Equation (11), Gauss's laws for the field $\psi=\left(\psi_{1}, \psi_{2}, \psi_{3}\right)^{\mathrm{T}}$ and the field $\psi=\left(\psi_{4}, \psi_{5}, \psi_{6}\right)^{\mathrm{T}}$ are written as follows:

$$
\begin{aligned}
& \frac{\partial \psi_{1}}{\partial x_{1}}+\frac{\partial \psi_{2}}{\partial x_{2}}+\frac{\partial \psi_{3}}{\partial x_{3}}=\rho_{1} \\
& \frac{\partial \psi_{4}}{\partial x_{1}}+\frac{\partial \psi_{5}}{\partial x_{2}}+\frac{\partial \psi_{6}}{\partial x_{3}}=\rho_{2}
\end{aligned}
$$

where $\rho_{1}$ and $\rho_{2}$ are physical quantities that can be identified with the electric and magnetic charge density. Using Equation (29) and Equations (17)-(19) then from Equations (23)-(25) we obtain the following system of equations:

$$
\begin{aligned}
& \frac{\partial^{2} \psi_{1}}{\partial t^{2}}+\frac{\partial^{2} \psi_{1}}{\partial x_{1}^{2}}+\frac{\partial^{2} \psi_{1}}{\partial x_{2}^{2}}+\frac{\partial^{2} \psi_{1}}{\partial x_{3}^{2}}+2 k_{1} \frac{\partial \psi_{1}}{\partial t} \\
& =-k_{1}^{2} \psi_{1}-k_{1} k_{2} j_{1}-k_{2}\left(\frac{\partial j_{1}}{\partial t}+\frac{\partial j_{3}}{\partial x_{2}}-\frac{\partial j_{2}}{\partial x_{3}}\right)+\frac{\partial \rho_{1}}{\partial x_{1}} \\
& \frac{\partial^{2} \psi_{2}}{\partial t^{2}}+\frac{\partial^{2} \psi_{2}}{\partial x_{1}^{2}}+\frac{\partial^{2} \psi_{2}}{\partial x_{2}^{2}}+\frac{\partial^{2} \psi_{2}}{\partial x_{3}^{2}}+2 k_{1} \frac{\partial \psi_{2}}{\partial t} \\
& =-k_{1}^{2} \psi_{2}-k_{1} k_{2} j_{2}-k_{2}\left(\frac{\partial j_{2}}{\partial t}+\frac{\partial j_{3}}{\partial x_{2}}-\frac{\partial j_{1}}{\partial x_{3}}\right)+\frac{\partial \rho_{1}}{\partial x_{2}} \\
& \frac{\partial^{2} \psi_{3}}{\partial t^{2}}+\frac{\partial^{2} \psi_{3}}{\partial x_{1}^{2}}+\frac{\partial^{2} \psi_{3}}{\partial x_{2}^{2}}+\frac{\partial^{2} \psi_{3}}{\partial x_{1}^{2}}+2 k_{1} \frac{\partial \psi_{3}}{\partial t} \\
& =-k_{1}^{2} \psi_{3}-k_{1} k_{2} j_{3}-k_{2}\left(\frac{\partial j_{3}}{\partial t}+\frac{\partial j_{2}}{\partial x_{1}}-\frac{\partial j_{1}}{\partial x_{2}}\right)+\frac{\partial \rho_{1}}{\partial x_{3}}
\end{aligned}
$$

In order to obtain a system of differential equations that can be applied to the electromagnetic field we set $k_{1}=0$. Then Equations (31)-(33) reduce to the following system of equations:

$$
\begin{aligned}
& \frac{\partial^{2} \psi_{1}}{\partial t^{2}}+\frac{\partial^{2} \psi_{1}}{\partial x_{1}^{2}}+\frac{\partial^{2} \psi_{1}}{\partial x_{2}^{2}}+\frac{\partial^{2} \psi_{1}}{\partial x_{3}^{2}}=-k_{2}\left(\frac{\partial j_{1}}{\partial t}+\frac{\partial j_{3}}{\partial x_{2}}-\frac{\partial j_{2}}{\partial x_{3}}\right)+\frac{\partial \rho_{1}}{\partial x_{1}} \\
& \frac{\partial^{2} \psi_{2}}{\partial t^{2}}+\frac{\partial^{2} \psi_{2}}{\partial x_{1}^{2}}+\frac{\partial^{2} \psi_{2}}{\partial x_{2}^{2}}+\frac{\partial^{2} \psi_{2}}{\partial x_{3}^{2}}=-k_{2}\left(\frac{\partial j_{2}}{\partial t}+\frac{\partial j_{3}}{\partial x_{2}}-\frac{\partial j_{1}}{\partial x_{3}}\right)+\frac{\partial \rho_{1}}{\partial x_{2}} \\
& \frac{\partial^{2} \psi_{3}}{\partial t^{2}}+\frac{\partial^{2} \psi_{3}}{\partial x_{1}^{2}}+\frac{\partial^{2} \psi_{3}}{\partial x_{2}^{2}}+\frac{\partial^{2} \psi_{3}}{\partial x_{1}^{2}}=-k_{2}\left(\frac{\partial j_{3}}{\partial t}+\frac{\partial j_{2}}{\partial x_{1}}-\frac{\partial j_{1}}{\partial x_{2}}\right)+\frac{\partial \rho_{1}}{\partial x_{3}}
\end{aligned}
$$

The equations given in Equations (34)-(36) are elliptic equations rather than hyperbolic or wave equations therefore these subfields are more suitable to rep- 
resent stable quantum particles with invariant physical properties. Moreover, since elliptic equations comply with the Euclidean relativity instead of the pseudoEuclidean relativity therefore there may exist some physical properties associated with quantum particles that can travel with speeds greater than the speed of light in vacuum, which is a speed limit of transmission for physical events that comply with the pseudo-Euclidean relativity.

Similarly, by using the matrices $A_{i}$ given in Equation (13), Equations (26)-(28), Gauss's laws given in Equation (30), and $k_{1}=0$, a system of equations with the positive sign for the matrix $A_{0}$ can be obtained and given as follows:

$$
\begin{aligned}
& \frac{\partial^{2} \psi_{4}}{\partial t^{2}}+\frac{\partial^{2} \psi_{4}}{\partial x_{1}^{2}}+\frac{\partial^{2} \psi_{4}}{\partial x_{2}^{2}}+\frac{\partial^{2} \psi_{4}}{\partial x_{3}^{2}}=-k_{2}\left(-\frac{\partial j_{4}}{\partial t}+\frac{\partial j_{6}}{\partial x_{2}}-\frac{\partial j_{5}}{\partial x_{3}}\right)+\frac{\partial \rho_{2}}{\partial x_{1}} \\
& \frac{\partial^{2} \psi_{5}}{\partial t^{2}}+\frac{\partial^{2} \psi_{5}}{\partial x_{1}^{2}}+\frac{\partial^{2} \psi_{5}}{\partial x_{2}^{2}}+\frac{\partial^{2} \psi_{5}}{\partial x_{3}^{2}}=-k_{2}\left(-\frac{\partial j_{5}}{\partial t}+\frac{\partial j_{6}}{\partial x_{2}}-\frac{\partial j_{4}}{\partial x_{3}}\right)+\frac{\partial \rho_{2}}{\partial x_{2}} \\
& \frac{\partial^{2} \psi_{6}}{\partial t^{2}}+\frac{\partial^{2} \psi_{6}}{\partial x_{1}^{2}}+\frac{\partial^{2} \psi_{6}}{\partial x_{2}^{2}}+\frac{\partial^{2} \psi_{6}}{\partial x_{1}^{2}}=-k_{2}\left(-\frac{\partial j_{6}}{\partial t}+\frac{\partial j_{5}}{\partial x_{1}}-\frac{\partial j_{4}}{\partial x_{2}}\right)+\frac{\partial \rho_{2}}{\partial x_{3}}
\end{aligned}
$$

The equations of the subfield with the positive sign for the matrix $A_{0}$ also satisfy elliptic equations rather than wave equations therefore they are also suitable to represent quantum particles with stable properties that accompany the stable properties associated with the field equations given in Equations (37)-(39).

Having shown the basic equations for the two subfields by using the matrices $A_{i}$ with negative and positive time, each of which can be used to represent stable properties of quantum particles due to the fact that they satisfy elliptic equations rather than wave equations, we now show that a coupling of these two subfields can give rise to a coupled field that satisfies wave equations such as Maxwell field equations of the electromagnetic field. A coupled field from the two subfields with the matrices given in Equation (13) can be formulated by using the following coupled matrices.

$$
\begin{array}{ll}
A_{0} & =\left(\begin{array}{cccccc}
-1 & 0 & 0 & 0 & 0 & 0 \\
0 & -1 & 0 & 0 & 0 & 0 \\
0 & 0 & -1 & 0 & 0 & 0 \\
0 & 0 & 0 & 1 & 0 & 0 \\
0 & 0 & 0 & 0 & 1 & 0 \\
0 & 0 & 0 & 0 & 0 & 1
\end{array}\right), A_{1}=\left(\begin{array}{cccccc}
0 & 0 & 0 & 0 & 0 & 0 \\
0 & 0 & 0 & 0 & 0 & -1 \\
0 & 0 & 0 & 0 & 1 & 0 \\
0 & 0 & 0 & 0 & 0 & 0 \\
0 & 0 & -1 & 0 & 0 & 0 \\
0 & 1 & 0 & 0 & 0 & 0
\end{array}\right), \\
A_{2} & =\left(\begin{array}{cccccc}
0 & 0 & 0 & 0 & 0 & 1 \\
0 & 0 & 0 & 0 & 0 & 0 \\
0 & 0 & 0 & -1 & 0 & 0 \\
0 & 0 & 1 & 0 & 0 & 0 \\
0 & 0 & 0 & 0 & 0 & 0 \\
-1 & 0 & 0 & 0 & 0 & 0
\end{array}\right), A_{3}=\left(\begin{array}{cccccc}
0 & 0 & 0 & 0 & -1 & 0 \\
0 & 0 & 0 & 1 & 0 & 0 \\
0 & 0 & 0 & 0 & 0 & 0 \\
0 & -1 & 0 & 0 & 0 & 0 \\
1 & 0 & 0 & 0 & 0 & 0 \\
0 & 0 & 0 & 0 & 0 & 0
\end{array}\right)
\end{array}
$$

Then we obtain the following results: 


$$
\begin{aligned}
& A_{0}^{2}=\left(\begin{array}{cccccc}
1 & 0 & 0 & 0 & 0 & 0 \\
0 & 1 & 0 & 0 & 0 & 0 \\
0 & 0 & 1 & 0 & 0 & 0 \\
0 & 0 & 0 & 1 & 0 & 0 \\
0 & 0 & 0 & 0 & 1 & 0 \\
0 & 0 & 0 & 0 & 0 & 1
\end{array}\right), \quad A_{1}^{2}=\left(\begin{array}{cccccc}
0 & 0 & 0 & 0 & 0 & 0 \\
0 & -1 & 0 & 0 & 0 & 0 \\
0 & 0 & -1 & 0 & 0 & 0 \\
0 & 0 & 0 & 0 & 0 & 0 \\
0 & 0 & 0 & 0 & -1 & 0 \\
0 & 0 & 0 & 0 & 0 & -1
\end{array}\right) \\
& A_{2}^{2}=\left(\begin{array}{cccccc}
-1 & 0 & 0 & 0 & 0 & 0 \\
0 & 0 & 0 & 0 & 0 & 0 \\
0 & 0 & -1 & 0 & 0 & 0 \\
0 & 0 & 0 & -1 & 0 & 0 \\
0 & 0 & 0 & 0 & 0 & 0 \\
0 & 0 & 0 & 0 & 0 & -1
\end{array}\right), A_{3}^{2}=\left(\begin{array}{cccccc}
-1 & 0 & 0 & 0 & 0 & 0 \\
0 & -1 & 0 & 0 & 0 & 0 \\
0 & 0 & 0 & 0 & 0 & 0 \\
0 & 0 & 0 & -1 & 0 & 0 \\
0 & 0 & 0 & 0 & -1 & 0 \\
0 & 0 & 0 & 0 & 0 & 0
\end{array}\right) \\
& A_{1} A_{2}+A_{2} A_{1}=\left(\begin{array}{cccccc}
0 & 1 & 0 & 0 & 0 & 0 \\
1 & 0 & 0 & 0 & 0 & 0 \\
0 & 0 & 0 & 0 & 0 & 0 \\
0 & 0 & 0 & 0 & 1 & 0 \\
0 & 0 & 0 & 1 & 0 & 0 \\
0 & 0 & 0 & 0 & 0 & 0
\end{array}\right), \quad A_{1} A_{3}+A_{3} A_{1}=\left(\begin{array}{cccccc}
0 & 0 & 1 & 0 & 0 & 0 \\
0 & 0 & 0 & 0 & 0 & 0 \\
1 & 0 & 0 & 0 & 0 & 0 \\
0 & 0 & 0 & 0 & 0 & 1 \\
0 & 0 & 0 & 0 & 0 & 0 \\
0 & 0 & 0 & 1 & 0 & 0
\end{array}\right) \\
& A_{2} A_{3}+A_{3} A_{2}=\left(\begin{array}{cccccc}
0 & 0 & 0 & 0 & 0 & 0 \\
0 & 0 & 1 & 0 & 0 & 0 \\
0 & 1 & 0 & 0 & 0 & 0 \\
0 & 0 & 0 & 0 & 0 & 0 \\
0 & 0 & 0 & 0 & 0 & 1 \\
0 & 0 & 0 & 0 & 1 & 0
\end{array}\right), \quad A_{0} A_{i}+A_{i} A_{0}=0 \text { for } i=1,2,3
\end{aligned}
$$

It is noticed from the results obtained in Equation (41) that by coupling the two subfields with negative and positive time the cross derivatives that involve time are automatically removed. This shows that the electromagnetic field may be considered as a resonant field which is formed from the superposition of two physical fields that flow in opposite temporal directions. Similar to the case of subfields, we also rewrite Equation (7) for the coupled field in the following simple form:

$$
\left(A_{0} \frac{\partial}{\partial t}+A_{1} \frac{\partial}{\partial x_{1}}+A_{2} \frac{\partial}{\partial x_{2}}+A_{3} \frac{\partial}{\partial x_{3}}\right) \psi=k_{1} \psi+k_{2} J
$$

where $\psi=\left(\psi_{1}, \psi_{2}, \psi_{3}, \psi_{4}, \psi_{5}, \psi_{6}\right)^{\mathrm{T}}$ and $J=\left(j_{1}, j_{2}, j_{3}, j_{4}, j_{5}, j_{6}\right)^{\mathrm{T}}$. Using the matrices given in Equation (40) we obtain the following system of equations for the coupled field from Equation (42):

$$
\begin{aligned}
& -\frac{\partial \psi_{1}}{\partial t}+\frac{\partial \psi_{6}}{\partial x_{2}}-\frac{\partial \psi_{5}}{\partial x_{3}}=k_{1} \psi_{1}+k_{2} j_{1} \\
& -\frac{\partial \psi_{2}}{\partial t}+\frac{\partial \psi_{4}}{\partial x_{3}}-\frac{\partial \psi_{6}}{\partial x_{1}}=k_{1} \psi_{2}+k_{2} j_{2}
\end{aligned}
$$




$$
\begin{gathered}
-\frac{\partial \psi_{3}}{\partial t}+\frac{\partial \psi_{5}}{\partial x_{1}}-\frac{\partial \psi_{4}}{\partial x_{2}}=k_{1} \psi_{3}+k_{2} j_{3} \\
\frac{\partial \psi_{4}}{\partial t}+\frac{\partial \psi_{3}}{\partial x_{2}}-\frac{\partial \psi_{2}}{\partial x_{3}}=k_{1} \psi_{4}+k_{2} j_{4} \\
\frac{\partial \psi_{5}}{\partial t}+\frac{\partial \psi_{1}}{\partial x_{3}}-\frac{\partial \psi_{3}}{\partial x_{1}}=k_{1} \psi_{5}+k_{2} j_{5} \\
\frac{\partial \psi_{6}}{\partial t}+\frac{\partial \psi_{2}}{\partial x_{1}}-\frac{\partial \psi_{1}}{\partial x_{2}}=k_{1} \psi_{6}+k_{2} j_{6}
\end{gathered}
$$

Using the results obtained for the matrices $A_{i}$ given in Equation (41) we obtain the following system of equations for the coupled field from Equation (9):

$$
\begin{aligned}
& \frac{\partial^{2} \psi_{1}}{\partial t^{2}}-\frac{\partial^{2} \psi_{1}}{\partial x_{2}^{2}}-\frac{\partial^{2} \psi_{1}}{\partial x_{3}^{2}}+\frac{\partial}{\partial x_{1}}\left(\frac{\partial \psi_{2}}{\partial x_{2}}+\frac{\partial \psi_{3}}{\partial x_{3}}\right) \\
& =k_{1}^{2} \psi_{1}+k_{1} k_{2} j_{1}+k_{2}\left(-\frac{\partial j_{1}}{\partial t}+\frac{\partial j_{6}}{\partial x_{2}}-\frac{\partial j_{5}}{\partial x_{3}}\right) \\
& \frac{\partial^{2} \psi_{2}}{\partial t^{2}}-\frac{\partial^{2} \psi_{2}}{\partial x_{1}^{2}}-\frac{\partial^{2} \psi_{2}}{\partial x_{3}^{2}}+\frac{\partial}{\partial x_{2}}\left(\frac{\partial \psi_{1}}{\partial x_{1}}+\frac{\partial \psi_{3}}{\partial x_{3}}\right) \\
& =k_{1}^{2} \psi_{2}+k_{1} k_{2} j_{2}+k_{2}\left(-\frac{\partial j_{2}}{\partial t}+\frac{\partial j_{4}}{\partial x_{3}}-\frac{\partial j_{6}}{\partial x_{1}}\right) \\
& \frac{\partial^{2} \psi_{3}}{\partial t^{2}}-\frac{\partial^{2} \psi_{3}}{\partial x_{1}^{2}}-\frac{\partial^{2} \psi_{3}}{\partial x_{2}^{2}}+\frac{\partial}{\partial x_{3}}\left(\frac{\partial \psi_{1}}{\partial x_{1}}+\frac{\partial \psi_{2}}{\partial x_{2}}\right) \\
& =k_{1}^{2} \psi_{3}+k_{1} k_{2} j_{3}+k_{2}\left(-\frac{\partial j_{3}}{\partial t}+\frac{\partial j_{5}}{\partial x_{1}}-\frac{\partial j_{4}}{\partial x_{2}}\right) \\
& \frac{\partial^{2} \psi_{4}}{\partial t^{2}}-\frac{\partial^{2} \psi_{4}}{\partial x_{2}^{2}}-\frac{\partial^{2} \psi_{4}}{\partial x_{3}^{2}}+\frac{\partial}{\partial x_{1}}\left(\frac{\partial \psi_{5}}{\partial x_{2}}+\frac{\partial \psi_{6}}{\partial x_{3}}\right) \\
& =k_{1}^{2} \psi_{4}+k_{1} k_{2} j_{4}+k_{2}\left(\frac{\partial j_{4}}{\partial t}+\frac{\partial j_{3}}{\partial x_{2}}-\frac{\partial j_{2}}{\partial x_{3}}\right) \\
& \frac{\partial^{2} \psi_{5}}{\partial t^{2}}-\frac{\partial^{2} \psi_{5}}{\partial x_{1}^{2}}-\frac{\partial^{2} \psi_{5}}{\partial x_{3}^{2}}+\frac{\partial}{\partial x_{2}}\left(\frac{\partial \psi_{4}}{\partial x_{1}}+\frac{\partial \psi_{6}}{\partial x_{3}}\right) \\
& =k_{1}^{2} \psi_{5}+k_{1} k_{2} j_{5}+k_{2}\left(\frac{\partial j_{5}}{\partial t}+\frac{\partial j_{1}}{\partial x_{3}}-\frac{\partial j_{3}}{\partial x_{1}}\right) \\
& \frac{\partial^{2} \psi_{6}}{\partial t^{2}}-\frac{\partial^{2} \psi_{6}}{\partial x_{1}^{2}}-\frac{\partial^{2} \psi_{6}}{\partial x_{2}^{2}}+\frac{\partial}{\partial x_{3}}\left(\frac{\partial \psi_{4}}{\partial x_{1}}+\frac{\partial \psi_{5}}{\partial x_{2}}\right) \\
& =k_{1}^{2} \psi_{6}+k_{1} k_{2} j_{6}+k_{2}\left(\frac{\partial j_{6}}{\partial t}+\frac{\partial j_{2}}{\partial x_{1}}-\frac{\partial j_{1}}{\partial x_{2}}\right)
\end{aligned}
$$

Using the divergence conditions or Gauss's laws given in Equations ((29), (30)) the system of equations given in Equations (49)-(54) reduces to the following system of equations: 


$$
\begin{aligned}
& \frac{\partial^{2} \psi_{1}}{\partial t^{2}}-\frac{\partial^{2} \psi_{1}}{\partial x_{1}^{2}}-\frac{\partial^{2} \psi_{1}}{\partial x_{2}^{2}}-\frac{\partial^{2} \psi_{1}}{\partial x_{3}^{2}} \\
& =k_{1}^{2} \psi_{1}+k_{1} k_{2} j_{1}+k_{2}\left(-\frac{\partial j_{1}}{\partial t}+\frac{\partial j_{6}}{\partial x_{2}}-\frac{\partial j_{5}}{\partial x_{3}}\right)-\frac{\partial \rho_{1}}{\partial x_{1}} \\
& \frac{\partial^{2} \psi_{2}}{\partial t^{2}}-\frac{\partial^{2} \psi_{2}}{\partial x_{1}^{2}}-\frac{\partial^{2} \psi_{2}}{\partial x_{2}^{2}}-\frac{\partial^{2} \psi_{2}}{\partial x_{3}^{2}} \\
& =k_{1}^{2} \psi_{2}+k_{1} k_{2} j_{2}+k_{2}\left(-\frac{\partial j_{2}}{\partial t}+\frac{\partial j_{4}}{\partial x_{3}}-\frac{\partial j_{6}}{\partial x_{1}}\right)-\frac{\partial \rho_{1}}{\partial x_{2}} \\
& \frac{\partial^{2} \psi_{3}}{\partial t^{2}}-\frac{\partial^{2} \psi_{3}}{\partial x_{1}^{2}}-\frac{\partial^{2} \psi_{3}}{\partial x_{2}^{2}}-\frac{\partial^{2} \psi_{3}}{\partial x_{3}^{2}} \\
& =k_{1}^{2} \psi_{3}+k_{1} k_{2} j_{3}+k_{2}\left(-\frac{\partial j_{3}}{\partial t}+\frac{\partial j_{5}}{\partial x_{1}}-\frac{\partial j_{4}}{\partial x_{2}}\right)-\frac{\partial \rho_{1}}{\partial x_{3}} \\
& \frac{\partial^{2} \psi_{4}}{\partial t^{2}}-\frac{\partial^{2} \psi_{4}}{\partial x_{1}^{2}}-\frac{\partial^{2} \psi_{4}}{\partial x_{2}^{2}}-\frac{\partial^{2} \psi_{4}}{\partial x_{3}^{2}} \\
& =k_{1}^{2} \psi_{4}+k_{1} k_{2} j_{4}+k_{2}\left(\frac{\partial j_{4}}{\partial t}+\frac{\partial j_{3}}{\partial x_{2}}-\frac{\partial j_{2}}{\partial x_{3}}\right)-\frac{\partial \rho_{2}}{\partial x_{1}} \\
& \frac{\partial^{2} \psi_{5}}{\partial t^{2}}-\frac{\partial^{2} \psi_{5}}{\partial x_{1}^{2}}-\frac{\partial^{2} \psi_{5}}{\partial x_{2}^{2}}-\frac{\partial^{2} \psi_{5}}{\partial x_{3}^{2}} \\
& =k_{1}^{2} \psi_{5}+k_{1} k_{2} j_{5}+k_{2}\left(\frac{\partial j_{5}}{\partial t}+\frac{\partial j_{1}}{\partial x_{3}}-\frac{\partial j_{3}}{\partial x_{1}}\right)-\frac{\partial \rho_{2}}{\partial x_{2}} \\
& \frac{\partial^{2} \psi_{6}}{\partial t^{2}}-\frac{\partial^{2} \psi_{6}}{\partial x_{1}^{2}}-\frac{\partial^{2} \psi_{6}}{\partial x_{2}^{2}}-\frac{\partial^{2} \psi_{6}}{\partial x_{3}^{2}} \\
& =k_{1}^{2} \psi_{6}+k_{1} k_{2} j_{6}+k_{2}\left(\frac{\partial j_{6}}{\partial t}+\frac{\partial j_{2}}{\partial x_{1}}-\frac{\partial j_{1}}{\partial x_{2}}\right)-\frac{\partial \rho_{2}}{\partial x_{3}}
\end{aligned}
$$

Now, to obtain Maxwell field equations of the electromagnetic field we set $k_{1}=0$ and the system of equations given in Equations (43)-(48) reduces to:

$$
\begin{gathered}
-\frac{\partial \psi_{1}}{\partial t}+\frac{\partial \psi_{6}}{\partial x_{2}}-\frac{\partial \psi_{5}}{\partial x_{3}}=k_{2} j_{1} \\
-\frac{\partial \psi_{2}}{\partial t}+\frac{\partial \psi_{4}}{\partial x_{3}}-\frac{\partial \psi_{6}}{\partial x_{1}}=k_{2} j_{2} \\
-\frac{\partial \psi_{3}}{\partial t}+\frac{\partial \psi_{5}}{\partial x_{1}}-\frac{\partial \psi_{4}}{\partial x_{2}}=k_{2} j_{3} \\
\frac{\partial \psi_{4}}{\partial t}+\frac{\partial \psi_{3}}{\partial x_{2}}-\frac{\partial \psi_{2}}{\partial x_{3}}=k_{2} j_{4} \\
\frac{\partial \psi_{5}}{\partial t}+\frac{\partial \psi_{1}}{\partial x_{3}}-\frac{\partial \psi_{3}}{\partial x_{1}}=k_{2} j_{5} \\
\frac{\partial \psi_{6}}{\partial t}+\frac{\partial \psi_{2}}{\partial x_{1}}-\frac{\partial \psi_{1}}{\partial x_{2}}=k_{2} j_{6}
\end{gathered}
$$


By identifying $\boldsymbol{E}=\left(\psi_{1}, \psi_{2}, \psi_{3}\right), \quad \boldsymbol{B}=\left(\psi_{4}, \psi_{5}, \psi_{6}\right), \quad \boldsymbol{j}_{1}=\left(j_{1}, j_{2}, j_{3}\right)$ and $\boldsymbol{j}_{2}=\left(j_{4}, j_{5}, j_{6}\right)$ the system of equations given in Equations (61)-(66), together with Gauss's laws given in Equations ((29), (30)), can be rewritten in the familiar form in classical electrodynamics:

$$
\begin{gathered}
\nabla \cdot \boldsymbol{E}=\rho_{1} \\
\nabla \cdot \boldsymbol{B}=\rho_{2} \\
\nabla \times \boldsymbol{E}+\frac{\partial \boldsymbol{B}}{\partial t}=k_{2} \boldsymbol{j}_{2} \\
\nabla \times \boldsymbol{B}-\frac{\partial \boldsymbol{E}}{\partial t}=k_{2} \boldsymbol{j}_{1}
\end{gathered}
$$

With $k_{1}=0$ we also obtain the following system of equations from Equations (55)-(60):

$$
\begin{aligned}
& \frac{\partial^{2} \psi_{1}}{\partial t^{2}}-\frac{\partial^{2} \psi_{1}}{\partial x_{1}^{2}}-\frac{\partial^{2} \psi_{1}}{\partial x_{2}^{2}}-\frac{\partial^{2} \psi_{1}}{\partial x_{3}^{2}}=k_{2}\left(-\frac{\partial j_{1}}{\partial t}+\frac{\partial j_{6}}{\partial x_{2}}-\frac{\partial j_{5}}{\partial x_{3}}\right)-\frac{\partial \rho_{1}}{\partial x_{1}} \\
& \frac{\partial^{2} \psi_{2}}{\partial t^{2}}-\frac{\partial^{2} \psi_{2}}{\partial x_{1}^{2}}-\frac{\partial^{2} \psi_{2}}{\partial x_{2}^{2}}-\frac{\partial^{2} \psi_{2}}{\partial x_{3}^{2}}=k_{2}\left(-\frac{\partial j_{2}}{\partial t}+\frac{\partial j_{4}}{\partial x_{3}}-\frac{\partial j_{6}}{\partial x_{1}}\right)-\frac{\partial \rho_{1}}{\partial x_{2}} \\
& \frac{\partial^{2} \psi_{3}}{\partial t^{2}}-\frac{\partial^{2} \psi_{3}}{\partial x_{1}^{2}}-\frac{\partial^{2} \psi_{3}}{\partial x_{2}^{2}}-\frac{\partial^{2} \psi_{3}}{\partial x_{3}^{2}}=k_{2}\left(-\frac{\partial j_{3}}{\partial t}+\frac{\partial j_{5}}{\partial x_{1}}-\frac{\partial j_{4}}{\partial x_{2}}\right)-\frac{\partial \rho_{1}}{\partial x_{3}} \\
& \frac{\partial^{2} \psi_{4}}{\partial t^{2}}-\frac{\partial^{2} \psi_{4}}{\partial x_{1}^{2}}-\frac{\partial^{2} \psi_{4}}{\partial x_{2}^{2}}-\frac{\partial^{2} \psi_{4}}{\partial x_{3}^{2}}=k_{2}\left(\frac{\partial j_{4}}{\partial t}+\frac{\partial j_{3}}{\partial x_{2}}-\frac{\partial j_{2}}{\partial x_{3}}\right)-\frac{\partial \rho_{2}}{\partial x_{1}} \\
& \frac{\partial^{2} \psi_{5}}{\partial t^{2}}-\frac{\partial^{2} \psi_{5}}{\partial x_{1}^{2}}-\frac{\partial^{2} \psi_{5}}{\partial x_{2}^{2}}-\frac{\partial^{2} \psi_{5}}{\partial x_{3}^{2}}=k_{2}\left(\frac{\partial j_{5}}{\partial t}+\frac{\partial j_{1}}{\partial x_{3}}-\frac{\partial j_{3}}{\partial x_{1}}\right)-\frac{\partial \rho_{2}}{\partial x_{2}} \\
& \frac{\partial^{2} \psi_{6}}{\partial t^{2}}-\frac{\partial^{2} \psi_{6}}{\partial x_{1}^{2}}-\frac{\partial^{2} \psi_{6}}{\partial x_{2}^{2}}-\frac{\partial^{2} \psi_{6}}{\partial x_{3}^{2}}=k_{2}\left(\frac{\partial j_{6}}{\partial t}+\frac{\partial j_{2}}{\partial x_{1}}-\frac{\partial j_{1}}{\partial x_{2}}\right)-\frac{\partial \rho_{2}}{\partial x_{3}}
\end{aligned}
$$

Equations (71)-(76) can be rewritten in a vector form as a system of two equations as in classical electrodynamics:

$$
\begin{aligned}
& \frac{\partial^{2} \boldsymbol{E}}{\partial t^{2}}-\nabla^{2} \boldsymbol{E}=\nabla\left(\rho_{1}\right)-k_{2} \frac{\partial \boldsymbol{J}_{e}}{\partial t}+k_{2} \nabla \times \boldsymbol{J}_{2} \\
& \frac{\partial^{2} \boldsymbol{B}}{\partial t^{2}}-\nabla^{2} \boldsymbol{B}=\nabla\left(\rho_{2}\right)-k_{2} \frac{\partial \boldsymbol{J}_{b}}{\partial t}+k_{2} \nabla \times \boldsymbol{J}_{1}
\end{aligned}
$$

where the charge density $\rho_{i}$ and the current density $\boldsymbol{j}_{i}$ satisfy the conservation law

$$
\nabla \cdot \boldsymbol{j}_{i}+\frac{\partial \rho_{i}}{\partial t}=0
$$

\subsection{Dirac Field as a Coupling of Two Elliptic Fields}

In this subsection, we will formulate Dirac field and subfields using the same procedure that we have applied to the Maxwell field of electromagnetism in the previous subsection. We have shown that Maxwell field is represented by matri- 
ces of rank six but the two subfields that are coupled to form Maxwell field are represented by matrices of rank three. Now, as it has been known that Dirac equation is formulated with matrices of rank four which are built upon Pauli matrices therefore we will simply use Pauli matrices as the required matrices for the two subfields. The Dirac equation then can be seen as a coupling of two systems of field equations similar to the case of Maxwell field equations of the electromagnetic field. Although the formulation of Dirac equation we consider in this work is straightforward from the known results there are new features that emerge with regard to the nature of the subfields that are coupled to form the Dirac field, such as the subfields also satisfy elliptic equations and therefore comply with the Euclidean relativity instead of wave equations and the pseudoEuclidean relativity. Except for the dimensions, these characteristics show that the quantum behaviours of both Maxwell and Dirac are similar when they are represented by the subfields. The Pauli matrices $A_{i}=\sigma_{i}$ that we use for Dirac subfields are given as follows:

$$
\begin{aligned}
& A_{0}=\mp\left(\begin{array}{ll}
1 & 0 \\
0 & 1
\end{array}\right), A_{1}=\left(\begin{array}{cc}
0 & 1 \\
1 & 0
\end{array}\right), \\
& A_{2}=\left(\begin{array}{cc}
0 & -i \\
i & 0
\end{array}\right), A_{3}=\left(\begin{array}{cc}
1 & 0 \\
0 & -1
\end{array}\right)
\end{aligned}
$$

We then obtain the following results:

$$
A_{i}^{2}=1 \text { and } A_{i} A_{j}+A_{j} A_{i}=0 \text { for } i, j=0,1,2,3
$$

Using the Pauli matrices $A_{i}$ given in Equation (80) with negative time we obtain the following system of differential equations from Equation (7):

$$
\begin{aligned}
& -\frac{\partial \psi_{1}}{\partial t}+\frac{\partial \psi_{2}}{\partial x_{1}}-i \frac{\partial \psi_{2}}{\partial x_{2}}+\frac{\partial \psi_{1}}{\partial x_{3}}=k_{1} \psi_{1}+k_{2} j_{1} \\
& -\frac{\partial \psi_{2}}{\partial t}+\frac{\partial \psi_{1}}{\partial x_{1}}+i \frac{\partial \psi_{1}}{\partial x_{2}}-\frac{\partial \psi_{2}}{\partial x_{3}}=k_{1} \psi_{2}+k_{2} j_{2}
\end{aligned}
$$

Using the Pauli matrices $A_{i}$ given in Equation (80) with positive time we obtain the following system of differential equations from Equation (7):

$$
\begin{aligned}
& \frac{\partial \psi_{3}}{\partial t}+\frac{\partial \psi_{4}}{\partial x_{1}}-i \frac{\partial \psi_{4}}{\partial x_{2}}+\frac{\partial \psi_{3}}{\partial x_{3}}=k_{1} \psi_{3}+k_{2} j_{1} \\
& \frac{\partial \psi_{4}}{\partial t}+\frac{\partial \psi_{3}}{\partial x_{1}}+i \frac{\partial \psi_{3}}{\partial x_{2}}-\frac{\partial \psi_{4}}{\partial x_{3}}=k_{1} \psi_{4}+k_{2} j_{2}
\end{aligned}
$$

On the other hand, using the results obtained in Equation (81) with negative time we obtain the following equation for the components of the function $\psi=\left(\psi_{1}, \psi_{2}\right)^{\mathrm{T}}$ from Equation (9):

$$
\begin{aligned}
& \frac{\partial^{2} \psi_{1}}{\partial t^{2}}+\frac{\partial^{2} \psi_{1}}{\partial x_{1}^{2}}+\frac{\partial^{2} \psi_{1}}{\partial x_{2}^{2}}+\frac{\partial^{2} \psi_{1}}{\partial x_{3}^{2}} \\
& =k_{1}^{2} \psi_{1}+k_{1} k_{2} j_{1}+k_{2}\left(-\frac{\partial j_{1}}{\partial t}+\frac{\partial j_{2}}{\partial x_{1}}-i \frac{\partial j_{2}}{\partial x_{2}}+\frac{\partial j_{1}}{\partial x_{3}}\right)
\end{aligned}
$$




$$
\begin{aligned}
& \frac{\partial^{2} \psi_{2}}{\partial t^{2}}+\frac{\partial^{2} \psi_{2}}{\partial x_{1}^{2}}+\frac{\partial^{2} \psi_{2}}{\partial x_{2}^{2}}+\frac{\partial^{2} \psi_{2}}{\partial x_{3}^{2}} \\
& =k_{1}^{2} \psi_{2}+k_{1} k_{2} j_{2}+k_{2}\left(-\frac{\partial j_{2}}{\partial t}+\frac{\partial j_{1}}{\partial x_{1}}+i \frac{\partial j_{1}}{\partial x_{2}}-\frac{\partial j_{2}}{\partial x_{3}}\right)
\end{aligned}
$$

Similarly, using the results obtained in Equation (81) with positive time we obtain the following equation for the components of the function $\psi=\left(\psi_{3}, \psi_{4}\right)^{\mathrm{T}}$ from Equation (9):

$$
\begin{aligned}
& \frac{\partial^{2} \psi_{3}}{\partial t^{2}}+\frac{\partial^{2} \psi_{3}}{\partial x_{1}^{2}}+\frac{\partial^{2} \psi_{3}}{\partial x_{2}^{2}}+\frac{\partial^{2} \psi_{3}}{\partial x_{3}^{2}} \\
& =k_{1}^{2} \psi_{3}+k_{1} k_{2} j_{3}+k_{2}\left(\frac{\partial j_{3}}{\partial t}+\frac{\partial j_{4}}{\partial x_{1}}-i \frac{\partial j_{4}}{\partial x_{2}}+\frac{\partial j_{3}}{\partial x_{3}}\right) \\
& \frac{\partial^{2} \psi_{4}}{\partial t^{2}}+\frac{\partial^{2} \psi_{4}}{\partial x_{1}^{2}}+\frac{\partial^{2} \psi_{4}}{\partial x_{2}^{2}}+\frac{\partial^{2} \psi_{4}}{\partial x_{3}^{2}} \\
& =k_{1}^{2} \psi_{4}+k_{1} k_{2} j_{4}+k_{2}\left(\frac{\partial j_{4}}{\partial t}+\frac{\partial j_{3}}{\partial x_{1}}+i \frac{\partial j_{3}}{\partial x_{2}}-\frac{\partial j_{4}}{\partial x_{3}}\right)
\end{aligned}
$$

As in the case of the subfields of Maxwell field of the electromagnetic field, the equations given in Equations (86)-(89) are elliptic equations therefore they can be used to describe the steady states of physical systems, in particular they can be used to explain the stability of elementary particles. Furthermore, if quantum particles possess physical properties that are represented by subfields which are described by elliptic equations, hence complying with the Euclidean relativity, then they can be used to explain physical phenomena that require physical transmissions with speeds greater than the speed of light in vacuum, such as the Einstein-Podosky-Rosen paradox in quantum entanglement.

Now, as being well-known the coupled field which can be used to represent Dirac field is formulated by using the familiar gamma matrices $\gamma^{\mu}$ written in terms of the Pauli and unit matrices as:

$$
\begin{gathered}
\gamma_{0}=\left(\begin{array}{cc}
I & 0 \\
0 & -I
\end{array}\right), \gamma_{i}=\left(\begin{array}{cc}
0 & \sigma_{i} \\
-\sigma_{i} & 0
\end{array}\right) \\
\gamma_{0}=\left(\begin{array}{cccc}
1 & 0 & 0 & 0 \\
0 & 1 & 0 & 0 \\
0 & 0 & -1 & 0 \\
0 & 0 & 0 & -1
\end{array}\right), \gamma_{1}=\left(\begin{array}{cccc}
0 & 0 & 0 & 1 \\
0 & 0 & 1 & 0 \\
0 & -1 & 0 & 0 \\
-1 & 0 & 0 & 0
\end{array}\right), \\
\gamma_{2}=\left(\begin{array}{cccc}
0 & 0 & 0 & -i \\
0 & 0 & i & 0 \\
0 & i & 0 & 0 \\
-i & 0 & 0 & 0
\end{array}\right), \gamma_{3}=\left(\begin{array}{cccc}
0 & 0 & 1 & 0 \\
0 & 0 & 0 & -1 \\
-1 & 0 & 0 & 0 \\
0 & 1 & 0 & 0
\end{array}\right)
\end{gathered}
$$

With $k_{2}=0$, Equation (7) reduces to Dirac equation for a free particle which is written in the form:

$$
\gamma^{\mu} \partial_{\mu} \psi=-i m \psi
$$


Using the gamma matrices given in Equation (91), Dirac equation given in Equation (92) can be written out for the wavefunction $\psi=\left(\psi_{1}, \psi_{2}, \psi_{3}, \psi_{4}\right)^{\mathrm{T}}$ as

$$
\begin{gathered}
\frac{\partial \psi_{1}}{\partial t}+i m \psi_{1}=-\frac{\partial \psi_{3}}{\partial z}-\left(\frac{\partial}{\partial x}+i \frac{\partial}{\partial y}\right) \psi_{4} \\
\frac{\partial \psi_{2}}{\partial t}+i m \psi_{2}=-\left(\frac{\partial}{\partial x}+i \frac{\partial}{\partial y}\right) \psi_{3}+\frac{\partial \psi_{4}}{\partial z} \\
\frac{\partial \psi_{3}}{\partial t}-i m \psi_{3}=-\frac{\partial \psi_{1}}{\partial z}-\left(\frac{\partial}{\partial x}-i \frac{\partial}{\partial y}\right) \psi_{2} \\
\frac{\partial \psi_{4}}{\partial t}-i m \psi_{4}=-\left(\frac{\partial}{\partial x}+i \frac{\partial}{\partial y}\right) \psi_{1}-\frac{\partial \psi_{2}}{\partial z}
\end{gathered}
$$

Dirac equation written as a system of linear first order partial differential equations given in Equations (93)-(96) suggests that matter wave can be interpreted as a coupling of two different physical subfields represented by the field $\left(\psi_{1}, \psi_{2}\right)$ and the field $\left(\psi_{3}, \psi_{4}\right)$ whose temporal rates of change will convert one field to the other. From the gamma matrices given in Equation (91) we obtain the following relations:

$$
\gamma_{0}^{2}=1, \quad \gamma_{i}^{2}=-1 \text { for } i=1,2,3 \text { and } \gamma_{i} \gamma_{j}+\gamma_{j} \gamma_{i}=0 \text { for } i \neq j
$$

With the relations obtained in Equation (97), it can be shown that all components of Dirac wavefunction $\psi=\left(\psi_{1}, \psi_{2}, \psi_{3}, \psi_{4}\right)^{\mathrm{T}}$ satisfy the Klein-Gordon equation:

$$
\frac{\partial^{2} \psi_{\mu}}{\partial t^{2}}-\frac{\partial^{2} \psi_{\mu}}{\partial x^{2}}-\frac{\partial^{2} \psi_{\mu}}{\partial y^{2}}-\frac{\partial^{2} \psi_{\mu}}{\partial z^{2}}=-m^{2} \psi_{\mu}
$$

The Klein-Gordon equation is a wave equation that is Lorentz invariant in the pseudo-Euclidean space which was proposed and developed by Minkowski based on Einstein's theory of special relativity.

In fact, it is possible to formulate a coupled field that is similar to Dirac field from the subfields represented by the Pauli matrices but instead satisfies an elliptic equation rather than a wave equation. Such field therefore will be Euclidean invariant. Consider a coupled field that is formed from the subfields represented by Pauli matrices with the coupled matrices given as follows:

$$
A_{0}=\left(\begin{array}{cc}
-I & 0 \\
0 & I
\end{array}\right), A_{i}=\left(\begin{array}{cc}
0 & \sigma_{i} \\
\sigma_{i} & 0
\end{array}\right)
$$

Then we obtain the following results:

$$
\gamma_{0}^{2}=1, \gamma_{i}^{2}=1 \text { for } i=1,2,3 \text { and } \gamma_{i} \gamma_{j}+\gamma_{j} \gamma_{i}=0 \text { for } i \neq j
$$

From the relations obtained in Equation (100), then it can be shown that all components of the wavefunction $\psi=\left(\psi_{1}, \psi_{2}, \psi_{3}, \psi_{4}\right)^{\mathrm{T}}$ satisfy the following elliptic equation:

$$
\frac{\partial^{2} \psi_{\mu}}{\partial t^{2}}+\frac{\partial^{2} \psi_{\mu}}{\partial x^{2}}+\frac{\partial^{2} \psi_{\mu}}{\partial y^{2}}+\frac{\partial^{2} \psi_{\mu}}{\partial z^{2}}=-m^{2} \psi_{\mu}
$$


As a further remark, we would like to mention here that we have formulated Maxwell and Dirac field essentially from a general system of linear first order partial differential equations which is a purely mathematical framework that can be used to formulate any physical theory that requires such mathematical structure, similar to the case of Laplace or Poisson's equation. Nonetheless, with such perspective, it has been suggested that they should be referred to as Maxwell-like and Dirac-like field equations instead of Maxwell and Dirac. The approach that we have used to formulate Maxwell and Dirac field is quite different from other mathematical methods such as gauge theories whose formulation is based on the variational principle [19] [20]. However, as we have shown in our work on the principle of least action that the variational principle with quantum objects may not lead to the least action as the principle is supposed to provide but only complies with Feynman's integral method of random paths or random surfaces, which itself is not related to the principle of least action [21]. Therefore, physical theories such as gauge theories that rely on the variational principle with a Lagrangian function to establish a deterministic least action should not be regarded as statistical theories therefore they are not in accordance with the current interpretation of the quantum theory which relies on the probability view for their interpretation of experimental results.

\section{A Classification of Relativity}

In this section, we show that relativity can be classified into three different types that are determined by the mathematical structures given to the spactime continuum so that it can manifest as three different types of relativistic spaces associated with the types of second order partial differential equations that are classified in Section 2. We call the spacetime continuum with the mathematical structure associated with the hyperbolic or wave equation a hyperbolic relativistic space and the corresponding relativity is the hyperbolic relativity. Similarly, we also define the elliptic and parabolic relativity. The problem that we address in this section is similar to our discussion on the fibre bundle structure of the spacetime continuum in which the spacetime continuum is the base space and all other physical events occur on different types of fibres and manifest in different physical forms that can be described by different mathematical structures [22]. We have shown that the spacetime structures result from different relationships between space and time and the apparent geometric and topological structures of the total spatiotemporal manifold are due to the dynamics and the geometric interactions of the decomposed cells from the base space of the total spatiotemporal manifold. The decomposed cells can form different types of fibres which may also geometrically interact with each other. In a more general context, we also discussed in detail a spacetime which has the mathematical structure of a 6-sphere bundle in which the dynamics of the fibres result from the geometric interactions of different types of decomposed cells that give rise to various relationships between space and time. In this case, it is assumed that we 
can only perceive within our physical ability the appearance of the grown intrinsic geometric structures on the base space of the total spatiotemporal manifold and the base space itself may not be observable with a reasonable assumption that a physical object is not observable if it does not have any form of geometric interactions. It could be that the base space of the spatiotemporal manifold at the beginning was only a six-dimensional Euclidean spatiotemporal continuum $R^{6}$ which had no non-trivial geometric structures therefore contained no physical objects. As we have shown in our work on Maxwell and Dirac field with threedimensional time [23], we can assume that the spatiotemporal manifold is described by a six-dimensional differentiable manifold $M$ which is composed of a three-dimensional spatial manifold and a three-dimensional temporal manifold, in which all physical objects are embedded, then the manifold $M$ can be decomposed in the form $M=M \# S_{S}^{3} \# S_{T}^{3}$, where $S_{S}^{3}$ and $S_{T}^{3}$ are the spatial and temporal 3-sphere, respectively. It is expected that the mathematical formulation of possible fibres of the spatiotemporal manifold should be derived from a general line element $\mathrm{d} s^{2}=g_{\alpha \beta} \mathrm{d} x^{\alpha} \mathrm{d} x^{\beta}$. In the following, however, we propose that the three types of relativity are classified in accordance with the classification of their corresponding coordinate transformations.

\subsection{Hyperbolic Relativity}

Hyperbolic relativity refers to the relativistic spacetime continuum with the mathematical structure of a pseudo-Euclidean space that associates with the hyperbolic type of the second order partial differential equations. In physics, the concept of a pseudo-Euclidean spacetime, or relativistic hyperbolic spacetime, was introduced by Minkowski in order to accommodate Einstein's theory of special relativity in which the coordinate transformation between the inertial frame $S$ with spacetime coordinates $(c t, x, y, z)$ and the inertial frame $S^{\prime}$ with coordinates $\left(c t^{\prime}, x^{\prime}, y^{\prime}, z^{\prime}\right)$ are derived from the principle of relativity and the postulate of a universal speed $c$, which is assumed to be the speed of light in vacuum. The coordinate transformation is the Lorentz transformation:

$$
\begin{aligned}
& x^{\prime}=\gamma(x-\beta c t), \quad y^{\prime}=y, \quad z^{\prime}=z, \\
& c t^{\prime}=\gamma(-\beta x+c t)
\end{aligned}
$$

where $\beta=v / c$ and $\gamma=1 / \sqrt{1-\beta^{2}}$. It can be shown that the Minkowski spacetime interval $c^{2} t^{2}-x^{2}-y^{2}-z^{2}$ is invariant under Lorentz transformation given in Equation (102). Now, in order to show that the hyperbolic equations are associated with the hyperbolic relativity, we need to show that the part that composes of the second order derivatives of the hyperbolic equation given in Equation (4) is invariant under Lorentz transformation. The Lorentz transformation and its inverse can be rewritten in the following forms:

$$
x^{\prime \mu}=\Lambda^{\mu}{ }_{\nu}{ }^{v} \text { and } x^{v}=\Lambda_{\mu}{ }^{v} x^{\prime \mu}
$$

where $x^{\mu}=(c t, x, y, z)$ and the Lorentz matrix $\Lambda^{\mu}{ }_{v}$ and its inverse $\Lambda_{\mu}{ }^{v}$ are given as: 


$$
\Lambda^{\mu}{ }_{\nu}=\left(\begin{array}{cccc}
\gamma & -\beta \gamma & 0 & 0 \\
-\beta \gamma & \gamma & 0 & 0 \\
0 & 0 & 1 & 0 \\
0 & 0 & 0 & 1
\end{array}\right) \text { and } \Lambda_{\mu}{ }^{\nu}=\left(\begin{array}{cccc}
\gamma & \beta \gamma & 0 & 0 \\
\beta \gamma & \gamma & 0 & 0 \\
0 & 0 & 1 & 0 \\
0 & 0 & 0 & 1
\end{array}\right)
$$

In order to show that the hyperbolic relativity associates with the hyperbolic type of second order partial differential equations we only need to show that the d'Alembert operator $\partial_{\mu} \partial^{\mu}=\partial^{2} / c^{2} \partial t^{2}-\nabla^{2}$ is invariant under Lorentz transformation, where the differential operators $\partial_{\mu}$ and $\partial^{\mu}$ are defined as $\partial_{\mu}=(\partial / c \partial t, \partial / \partial x, \partial / \partial y, \partial / \partial z)$ and $\partial^{\mu}=g^{\mu v} \partial_{v}=(-\partial / c \partial t, \partial / \partial x, \partial / \partial y, \partial / \partial z)^{\mathrm{T}}$. This can be seen by the fact that the differential operators $\partial_{\mu}$ and $\partial^{\mu}$ are vectors therefore by using the transformations $\partial_{\mu}^{\prime}=\Lambda_{\mu}{ }^{v} \partial_{v}$ and $\partial^{\prime \mu}=g^{\mu v} \partial_{v}^{\prime}$ we then obtain $\partial_{\mu}^{\prime} \partial^{\prime \mu}=\partial_{\mu} \partial^{\mu}$.

Now, it is remarkable that even though the concept of a relativistic hyperbolic spacetime originates from the invariance of Maxwell field equations under Lorentz transformation, Einstein was able to generalise it into a more general structure utilising the mathematics of differentiable manifold and the resulted theory has only been applied into the description of the gravitational field in which the electromagnetic field can only act as a source. And the only invariance that is required is the transformation of general coordinates. Einstein general relativity that complies with the hyperbolic relativity in curved spaces is represented in tensor form as $T_{\mu \nu}=k\left(R_{\mu \nu}-\frac{1}{2} R g_{\mu \nu}+\Lambda g_{\mu \nu}\right)$. Then using the centrally symmetric gravitational field with Schwarzschild metric:

$$
\mathrm{d} s^{2}=\mathrm{e}^{\psi} c^{2} \mathrm{~d} t^{2}-\mathrm{e}^{\chi} \mathrm{d} r^{2}-r^{2}\left(\mathrm{~d} \theta^{2}+\sin ^{2} \theta \mathrm{d} \phi^{2}\right)
$$

Schwarzschild solution can be found as:

$$
\mathrm{d} s^{2}=\left(1-\frac{C}{r}\right) c^{2} \mathrm{~d} t^{2}-\left(1-\frac{C}{r}\right)^{-1} \mathrm{~d} r^{2}-r^{2}\left(\mathrm{~d} \theta^{2}+\sin ^{2} \theta \mathrm{d} \phi^{2}\right)
$$

where $C=2 M G / c^{2}$. From the Schwarzschild solution, Newton law of gravity can be obtained as an approximation. We show in the next subsection on the elliptic relativistic spacetime that this result can also be obtained from the elliptic or Euclidean relativity.

\subsection{Elliptic Relativity}

Elliptic relativity refers to the relativistic spacetime continuum with the mathematical structure of a Euclidean space that associates with the elliptic type of the second order partial differential equations. We have also shown in our work on Euclidean relativity that it is possible to construct a special relativistic transformation that will make the four-dimensional spacetime continuum a Euclidean space rather than a pseudo-Euclidean space as in the case of Einstein's theory of special relativity. Consider the following modified Lorentz transformation:

$$
x^{\prime}=\gamma_{E}(x-\beta c t), y^{\prime}=y, \quad z^{\prime}=z, c t^{\prime}=\gamma_{E}(\beta x+c t)
$$


where $\beta=v / c$ and $\gamma_{E}$ will be determined from the principle of relativity and the postulate of a universal speed. If we now assume the invariance of the Euclidean interval $c^{2} t^{2}+x^{2}+y^{2}+z^{2}=c^{2} t^{\prime 2}+x^{\prime 2}+y^{\prime 2}+z^{\prime 2}$ then from the modified Lorentz transformation given in Equation (107), we obtain $\gamma_{E}=1 / \sqrt{1+\beta^{2}}$. It is seen from this expression for $\gamma_{E}$ that there is no upper limit in the relative speed $v$ between inertial frames. The value of $\gamma_{E}$ at the universal speed $v=C$ is $\gamma_{E}=1 / \sqrt{2}$. For the values of $v \ll c$, the modified Lorentz transformation also reduces to the Galilean transformation. However, it is interesting to observe that when $v \rightarrow \infty$ we have $\gamma_{E} \rightarrow 0$ and $\beta \gamma_{E} \rightarrow 1$, and in this case, we have $x^{\prime} \rightarrow-c t$ and $c t^{\prime} \rightarrow x$. This result shows that there is a conversion between space and time when $v \rightarrow \infty$. We can also derive the relativistic kinematics from the modified Lorentz transformation, such as the transformation of a length, the transformation of a time interval and the transformation of velocities. Let $L_{0}$ be the proper length and $\Delta t_{0}$ is the proper time interval then the length and the time interval transformations can be found as $L=\sqrt{1+\beta^{2}} L_{0}$ and $\Delta t=\Delta t_{0} / \sqrt{1+\beta^{2}}$. It is observed from the length transformation that the length of a moving object is expanding rather than contracting as in Einstein theory of special relativity. It is also observed from the time interval transformation that the proper time interval is longer than the same time interval measured by a moving observer. With the modified Lorentz transformation, the transformation of velocities can be found as:

$$
v_{x}^{\prime}=\frac{v_{x}-\beta c}{1+\beta v_{x} / c}, \quad v_{y}^{\prime}=\frac{v_{y}}{\gamma_{E}\left(1+\beta v_{x} / c\right)}, \quad v_{z}^{\prime}=\frac{v_{z}}{\gamma_{E}\left(1+\beta v_{x} / c\right)}
$$

From Equation (108), if we let $v_{x}=c$ then we obtain $v_{x}^{\prime}=((c-v) /(c+v)) c$. Therefore in this case $v_{x}^{\prime}=c$ only when the relative speed $v$ between two inertial frames vanishes. In other words, the universal speed $c$ is not the common speed of any moving physical object or physical field in inertial reference frames. In order to specify the nature of the assumed universal speed, we observe that in Einstein theory of special relativity it is assumed that spatial space of an inertial frame remains steady and this assumption is contradicted to Einstein theory of general relativity that shows that spatial space is actually expanding. Therefore it seems reasonable to suggest that the universal speed $c$ in the modified Lorentz transformation is the universal speed of expansion of the spatial space of all inertial frames. The modified Lorentz transformation and its inverse can be rewritten in the following forms:

$$
x^{\prime \mu}=\Lambda^{\mu}{ }_{\nu} x^{v} \text { and } x^{v}=\Lambda_{\mu}{ }^{v} x^{\prime \mu}
$$

where $x^{\mu}=(c t, x, y, z)$ and the modified Lorentz matrix $\Lambda^{\mu}{ }_{v}$ and its inverse $\Lambda_{\mu}{ }^{v}$ are given as:

$$
\Lambda_{\nu}^{\mu}=\left(\begin{array}{cccc}
\gamma_{E} & \beta \gamma_{E} & 0 & 0 \\
-\beta \gamma_{E} & \gamma_{E} & 0 & 0 \\
0 & 0 & 1 & 0 \\
0 & 0 & 0 & 1
\end{array}\right) \text { and } \Lambda_{\mu}{ }^{v}=\left(\begin{array}{cccc}
\gamma_{E} & -\beta \gamma_{E} & 0 & 0 \\
\beta \gamma_{E} & \gamma_{E} & 0 & 0 \\
0 & 0 & 1 & 0 \\
0 & 0 & 0 & 1
\end{array}\right)
$$


In order to show that the elliptic relativity associates with the elliptic type of second order partial differential equations we only need to show that the Euclidean differential operator $\partial_{\mu} \partial^{\mu}=\partial^{2} / c^{2} \partial t^{2}+\nabla^{2}$ is invariant under the modified Lorentz transformation given in Equation (20), where the differential operators $\partial_{\mu}$ and $\partial^{\mu}$ are defined as $\partial_{\mu}=(\partial / c \partial t, \partial / \partial x, \partial / \partial y, \partial / \partial z)$ and $\partial^{\mu}=g^{\mu v} \partial_{v}=(\partial / c \partial t, \partial / \partial x, \partial / \partial y, \partial / \partial z)^{\mathrm{T}}$. Since the differential operators $\partial_{\mu}$ and $\partial^{\mu}$ are vectors therefore by using the transformations $\partial_{\mu}^{\prime}=\Lambda_{\mu}{ }^{v} \partial_{\nu}$ and $\partial^{\prime \mu}=g^{\mu v} \partial_{v}^{\prime}$ we then obtain $\partial_{\mu}^{\prime} \partial^{\prime \mu}=\partial_{\mu} \partial^{\mu}$.

We assume that a general relativity that complies with the elliptic relativity in curved spaces can also be represented in tensor form as $T_{\mu v}=k\left(R_{\mu v}-\frac{1}{2} R g_{\mu v}+\Lambda g_{\mu v}\right)$. Then using the centrally symmetric gravitational field with Schwarzschild-like metric:

$$
\mathrm{d} s^{2}=\mathrm{e}^{\psi} c^{2} \mathrm{~d} t^{2}+\mathrm{e}^{\chi} \mathrm{d} r^{2}+r^{2}\left(\mathrm{~d} \theta^{2}+\sin ^{2} \theta \mathrm{d} \phi^{2}\right)
$$

Schwarzschild-like vacuum solution is found as:

$$
\mathrm{d} s^{2}=\left(1-\frac{C}{r}\right) c^{2} \mathrm{~d} t^{2}+\left(1-\frac{C}{r}\right)^{-1} \mathrm{~d} r^{2}+r^{2}\left(\mathrm{~d} \theta^{2}+\sin ^{2} \theta \mathrm{d} \phi^{2}\right)
$$

where $C=2 M G / c^{2}$. It can also be shown from the Schwarzschild-like solution given in Equation (112) that Newton law of gravity is obtained as an approximation [2].

\subsection{Parabolic Relativity}

We have shown that the hyperbolic and elliptic relativity are classified according to the mathematical structure of the second order derivatives of the second order partial differential equations $\sum_{i=1}^{n} \sum_{j=1}^{n} a_{i j} \partial^{2} \psi / \partial x_{i} \partial x_{j}$. For the hyperbolic relativity associated with the four-dimensional spacetime manifold we have

$\sum_{i=1}^{n} \sum_{j=1}^{n} a_{i j} \partial^{2} \psi / \partial x_{i} \partial x_{j}=\partial^{2} \psi / \partial t^{2}-\partial^{2} \psi / \partial x^{2}-\partial^{2} \psi / \partial y^{2}-\partial^{2} \psi / \partial z^{2}$. On the other hand, for the elliptic relativity we have

$\sum_{i=1}^{n} \sum_{j=1}^{n} a_{i j} \partial^{2} \psi / \partial x_{i} \partial x_{j}=\partial^{2} \psi / \partial t^{2}+\partial^{2} \psi / \partial x^{2}+\partial^{2} \psi / \partial y^{2}+\partial^{2} \psi / \partial z^{2}$. Now, for the case of the parabolic equation, because there are only three spatial components of second order derivatives for the four-dimensional spacetime continuum therefore as a consequence we consider the invariance of the parabolic equation only for these components under a parabolic coordinate transformation. Parabolic relativity refers to the relativistic spacetime continuum with the mathematical structure of a Euclidean space that associates with the parabolic type of the second order partial differential equations. There are many physical events that are described by the second order partial differential equations that involve only the spatial components of the second order derivatives therefore these physical events can be regarded as being associated with the parabolic relativity. In particular, the physical events that can be described by the diffusion equation and the Schrödinger equation that can be written generically as follows: 


$$
\frac{\partial^{2} \psi}{\partial x^{2}}+\frac{\partial^{2} \psi}{\partial y^{2}}+\frac{\partial^{2} \psi}{\partial z^{2}}=a \frac{\partial \psi}{\partial t}
$$

In Equation (113), we obtain the diffusion equation if $a \partial \psi / \partial t \neq 0$ and the Schrödinger equation by setting $a \partial \psi / \partial t=-(i 2 m / \hbar) \partial \psi / \partial t$. As in the case of the hyperbolic and elliptic relativity in which the Lorentz and modified Lorentz transformation involve only the second order derivatives of the differential equations, therefore to discuss coordinate transformation for the parabolic equation we should also consider the second order derivatives which form the Laplace operator. Therefore the corresponding transformations for the parabolic equation that leave the Laplace operator unchanged, that is in the parabolic relativity we only consider the invariance of the Euclidean spatial interval $x^{2}+y^{2}+z^{2}=x^{\prime 2}+y^{\prime 2}+z^{\prime 2}$. The time in parabolic relativity is therefore a universal time which is assumed to flow uniformly with the same rate in all reference systems. In general, the parabolic relativity is invariant with respect to the translation and rotation given as follows:

$$
x_{i}^{\prime}=x_{i}+a_{i} \text { and } x_{i}^{\prime}=\sum_{j=1}^{n} b_{i j} x_{j}
$$

where $\boldsymbol{x}=\left(x_{1}, x_{2}, \cdots, x_{n}\right)^{\mathrm{T}}$, and $A=\left\{a_{i}\right\}$ is a matrix for the translation and $B=\left\{b_{i j}\right\}$ is an orthogonal matrix for the rotation. If the matrix $B=\left\{b_{i j}\right\}$ is an orthogonal matrix then we have $\sum_{k=1}^{n} b_{i k} b_{j k}=\delta_{i}^{j}$, therefore we obtain the following result:

$$
\nabla_{x}^{2}=\sum_{i=1}^{n}\left(\sum_{j, k=1}^{n} b_{j i} b_{k i} \frac{\partial^{2}}{\partial x_{j}^{\prime} \partial x_{k}^{\prime}}\right)=\sum_{j, k=1}^{n}\left(\sum_{i=1}^{n} b_{j i} b_{k i}\right) \frac{\partial^{2}}{\partial x_{j}^{\prime} \partial x_{k}^{\prime}}=\nabla_{x^{\prime}}^{2}
$$

We now extend our discussion to a particular parabolic equation that is related to the curved structure of the spacetime manifold. We have shown in our work on the spacetime structure of quantum particles that they can be endowed with geometric and topological structures of differentiable manifolds and their motion should be described as isometric embeddings in higher Euclidean space that involve the diffusion equation. Fundamentally, we show that the three main dynamical descriptions of physical events in classical physics, namely Newton mechanics, Maxwell electromagnetism and Einstein gravitation, can be formulated in the same general covariant form and they can be represented by the general equation [8] [9]:

$$
\nabla_{\beta} M=k J
$$

where $M$ is a mathematical object that represents the corresponding physical system and $\nabla_{\beta}$ is a covariant derivative. For Newton mechanics,

$M=\frac{1}{2} m \sum_{\mu=1}^{3}\left(\mathrm{~d} x^{\mu} / \mathrm{d} t\right)^{2}+V$ and $J=0$. For Maxwell electromagnetism,

$M=F^{\alpha \beta}=\partial^{\mu} A^{v}-\partial^{v} A^{\mu}$, with the four-vector potential $A^{\mu} \equiv(V, \boldsymbol{A})$ and $J$ can be identified with the electric and magnetic currents. And for Einstein gravitation, $M=R^{\alpha \beta}$ and $J$ can be defined in terms of a metric $g_{\alpha \beta}$ and the Ricci 
scalar curvature using the Bianchi identities $\nabla_{\beta} R^{\alpha \beta}=\frac{1}{2} g^{\alpha \beta} \nabla_{\beta} R$, that is, $J=\frac{1}{2} g^{\alpha \beta} \nabla_{\beta} R$. If we use the Bianchi identities as field equations for the gravitational field then Einstein field equations $T_{\mu v}=k\left(R_{\mu v}-\frac{1}{2} R g_{\mu v}+\Lambda g_{\mu v}\right)$, as in the case of the electromagnetic field, should be regarded as a definition for the energy-momentum tensor $T_{\mu \nu}$ for the gravitational field. From the definition of the four-current $j^{\alpha}=\left(\rho, j_{i}\right)=\frac{1}{2} g^{\alpha \beta} \nabla_{\beta} R$ for the gravitational field, by comparing with the Poisson equation for a potential $V$ in classical physics, $\nabla^{2} V=4 \pi \rho$, we can identify the scalar potential $V$ with the Ricci scalar curvature $R$ and then obtain a diffusion equation:

$$
\frac{\partial R}{\partial t}=k\left(\frac{\partial^{2} R}{\partial x^{2}}+\frac{\partial^{2} R}{\partial y^{2}}+\frac{\partial^{2} R}{\partial z^{2}}\right)
$$

whose solutions can be found to take the form:

$$
R(x, y, z, t)=\left(\frac{M}{(\sqrt{4 \pi k t})^{3}}\right) \exp \left(-\frac{x^{2}+y^{2}+z^{2}}{4 k t}\right)
$$

which determines the probabilistic distribution of an amount of geometrical substance $M$ which is defined via the Ricci scalar curvature $R$ and manifests as observable matter. We have also shown that the Ricci scalar curvature $R$ associated with a differentiable manifold can be expressed in terms of the Schrödinger wavefunction $\psi$ in quantum mechanics. Now, instead of deriving a diffusion equation for the Ricci scalar curvature from the four-current we can also derive a diffusion equation for the Ricci scalar curvature from the Ricci flow by considering the case in which $J=\frac{1}{2} g^{\alpha \beta} \nabla_{\beta} R=0$. Then we obtain the equation:

$$
\nabla_{\beta} R^{\alpha \beta}=0
$$

Since $\nabla_{\mu} g^{\alpha \beta} \equiv 0$ for a given metric tensor $g^{\alpha \beta}$, Equation (119) implies $R^{\alpha \beta}=\Lambda g^{\alpha \beta}$ which can be written in a covariant form as:

$$
R_{\alpha \beta}=\Lambda g_{\alpha \beta}
$$

where $\Lambda$ is an undetermined constant. Using the identities $g_{\alpha \beta} g^{\alpha \beta}=4$ and $g_{\alpha \beta} R^{\alpha \beta}=R$, we obtain $\Lambda=R / 4$.

The Ricci flow can be derived from the field equation given in Equation (119) as follows [24] [25]. In differential geometry, the covariant derivative of a contravariant tensor of second rank $A^{\alpha \beta}$ is given by:

$$
\nabla_{\gamma} A^{\alpha \beta}=\partial_{\gamma} A^{\alpha \beta}+\Gamma_{\sigma \gamma}^{\alpha} A^{\sigma \beta}+\Gamma_{\sigma \gamma}^{\beta} A^{\alpha \sigma}
$$

The partial time derivative of Equation (121) is given as:

$$
\begin{aligned}
\partial_{t}\left(\nabla_{\gamma} A^{\alpha \beta}\right)= & \partial_{t}\left(\partial_{\gamma} A^{\alpha \beta}\right)+\left(\partial_{t} \Gamma_{\sigma \gamma}^{\alpha}\right) A^{\sigma \beta}+\Gamma_{\sigma \gamma}^{\alpha}\left(\partial_{t} A^{\sigma \beta}\right) \\
& +\left(\partial_{t} \Gamma_{\sigma \gamma}^{\beta}\right) A^{\alpha \sigma}+\Gamma_{\sigma \gamma}^{\beta}\left(\partial_{t} A^{\alpha \sigma}\right)
\end{aligned}
$$


Under the coordinate transformation $x^{\prime \alpha}=f^{\alpha}\left(x^{\beta}\right)$, the tensor $A^{\alpha \beta}$ is transformed as

$$
A^{\prime \alpha \beta}=\frac{\partial x^{\prime \alpha}}{\partial x^{\rho}} \frac{\partial x^{\prime \beta}}{\partial x^{\sigma}} A^{\rho \sigma}
$$

If the coordinate transformation is time-independent then the partial time derivative of the tensor $A^{\alpha \beta}$ is also a tensor which is transformed according to the rule:

$$
\frac{\partial A^{\prime \alpha \beta}}{\partial t}=\frac{\partial x^{\prime \alpha}}{\partial x^{\rho}} \frac{\partial x^{\prime \beta}}{\partial x^{\sigma}} \frac{\partial A^{\rho \sigma}}{\partial t}
$$

In this case, we have:

$$
\nabla_{\gamma}\left(\partial_{t} A^{\alpha \beta}\right)=\partial_{\gamma}\left(\partial_{t} A^{\alpha \beta}\right)+\Gamma_{\sigma \gamma}^{\alpha}\left(\partial_{t} A^{\sigma \beta}\right)+\Gamma_{\sigma \gamma}^{\beta}\left(\partial_{t} A^{\alpha \sigma}\right)
$$

It is observed from Equations (122) and (125) that if we impose the following condition on Equation (122):

$$
\left(\partial_{t} \Gamma_{\sigma \gamma}^{\alpha}\right) A^{\sigma \beta}+\left(\partial_{t} \Gamma_{\sigma \gamma}^{\beta}\right) A^{\alpha \sigma}=0
$$

then we obtain the identity:

$$
\nabla_{\gamma}\left(\partial_{t} A^{\alpha \beta}\right)=\partial_{t}\left(\nabla_{\gamma} A^{\alpha \beta}\right)
$$

In the case of a metric tensor $g^{\alpha \beta}$ then we have $\nabla_{\gamma}\left(\partial_{t} g^{\alpha \beta}\right)=\partial_{t}\left(\nabla_{\gamma} g^{\alpha \beta}\right) \equiv 0$, and from the field equations $\nabla_{\beta} R^{\alpha \beta}=0$ we arrive at the Ricci flow:

$$
\frac{\partial g_{\alpha \beta}}{\partial t}=k R_{\alpha \beta}
$$

From Equation (128) we can obtain a diffusion equation for the Ricci scalar curvature as follows [26] [27]:

$$
\frac{\partial R}{\partial t}=\Delta R+2 g^{\alpha \beta} g^{\gamma \sigma} R_{\alpha \sigma} R_{\beta \gamma}
$$

As a further remark, it should be mentioned here that it has been shown that parabolic equations have associated invariants that may be related to physical properties of physical objects. For example, consider a linear second order parabolic partial differential equation in two independent variables $x$ and $t$.

$$
\frac{\partial \psi}{\partial t}+a(x, t) \frac{\partial^{2} \psi}{\partial x^{2}}+b(x, t) \frac{\partial \psi}{\partial x}+c(x, t) \psi=0
$$

It is shown that the form of the parabolic equation given in Equation (130) is invariant under the group of equivalence transformations which consists of the linear transformation of the dependent variable and the invertible transformations of the independent variables as follows:

$$
\chi=\sigma(x, t) \psi, t^{\prime}=\varphi(t), x^{\prime}=\rho(x, t)
$$

where $\sigma(x, t), \varphi(t)$ and $\rho(x, t)$ are arbitrary functions [28] [29] [30]. The transformed equation of the equation given in Equation (131) then takes the form: 


$$
\frac{\partial \chi}{\partial t^{\prime}}+a^{\prime}\left(x^{\prime}, t^{\prime}\right) \frac{\partial^{2} \chi}{\partial x^{\prime 2}}+b^{\prime}\left(x^{\prime}, t^{\prime}\right) \frac{\partial \chi}{\partial x^{\prime}}+c^{\prime}\left(x^{\prime}, t^{\prime}\right) \chi=0
$$

Then an invariant of the parabolic equation given in Equation (130) is a function of the form

$$
J=J\left(a, b, c, \frac{\partial a}{\partial t}, \frac{\partial a}{\partial x}, \frac{\partial b}{\partial t}, \frac{\partial b}{\partial x}, \frac{\partial c}{\partial t}, \frac{\partial c}{\partial x}, \frac{\partial^{2} a}{\partial t^{2}}, \frac{\partial^{2} a}{\partial t \partial x}, \frac{\partial^{2} a}{\partial x^{2}}, \cdots\right) .
$$

\subsection{Simultaneous Relativities}

We have shown in previous subsections that the mathematical structure of the spacetime manifold can be classified in accordance with the classification of second order partial differential equations into hyperbolic, elliptic or parabolic relativistic space. On the other hand, normally a complex physical system such as an atom has various physical properties that involve different physical processes described by different types of second order partial differential equations in different relativities. If the physical properties associated with the physical system remain invariant then we can assume that they can be described independently by different second order partial differential equations, and hence their corresponding relativistic spaces should also exist independently from each other. As we have discussed in the introduction, these relativistic spaces may be considered as independent fibres of the spatiotemporal fibre bundle. Then in order to describe independent physical properties, we simply express all corresponding physical equations in all relativistic spaces simultaneously. For example, we assume that a physical system that possesses physical properties that can be described in the parabolic relativity and elliptic relativity respectively. If the parabolic property is massive and the elliptic property is massless then we have a simultaneous system of two equations that take the forms similar to the massive Schrödinger equation and massless Klein-Gordon equation as follows:

$$
\begin{gathered}
\frac{\partial^{2} \psi_{p}}{\partial x^{2}}+\frac{\partial^{2} \psi_{p}}{\partial y^{2}}+\frac{\partial^{2} \psi_{p}}{\partial z^{2}}=-i \frac{2 m}{\hbar} \frac{\partial \psi_{p}}{\partial t} \\
\frac{\partial^{2} \psi_{e}}{c_{e}^{2} \partial t^{2}}+\frac{\partial^{2} \psi_{e}}{\partial x_{1}^{2}}+\frac{\partial^{2} \psi_{e}}{\partial x_{2}^{2}}+\frac{\partial^{2} \psi_{e}}{\partial x_{3}^{2}}=0
\end{gathered}
$$

where $\psi_{p}$ and $\psi_{e}$ are wavefunctions in the parabolic and elliptic relativistic space respectively. We have also written $c_{e}$ to indicate that, unlike the universal speed $c$ in the hyperbolic relativity, the speed $c_{e}$ may be very large according to the elliptic relativity. In an $n$-dimensional space, solutions to Laplace equation can be expressed by the Green function as $G=k /\left(x_{1}^{2}+x_{2}^{2}+\cdots+x_{n}^{2}\right)^{\frac{n-2}{2}}$, hence for the Laplace equation given in Equation (135) with $n=4$, we obtain the solution:

$$
\psi_{e}=\frac{k}{c_{e}^{2} t^{2}+x^{2}+y^{2}+z^{2}}
$$

It is seen that if $c_{e}$ is very large then while other parabolic relativistic proper- 
ties of a quantum particle remain the same the elliptic properties vanish quickly with time, and in particular this result may be invoked to explain the EPR paradox in quantum mechanics. However, if different physical properties belong to the same type of relativity then we can express the total equation as a sum of different equations in the same relativistic space, as in the case we have shown in our work on spin dynamics that a total Schrödinger equation can be written as a sum of two separate Schrödinger equations in two different coordinate systems, one of them can be considered as intrinsic. This can be outlined as follows. Instead of introducing a spin operator, we introduce a differential operator that depends on an intrinsic coordinate system and can be used to formulate a spin dynamics. Since spin angular momentum and orbital angular momentum are similar in nature therefore it is possible to suggest that the spin operator in the intrinsic coordinate system should also have similar form to that of the orbital angular momentum operator. From this perspective, we can write a Schrödinger wave equation that is used to describe both the orbital and spin dynamics as follows [31]:

$$
\begin{aligned}
& -\frac{\hbar^{2}}{2 \mu} \nabla^{2} \Psi\left(\boldsymbol{r}, \boldsymbol{r}_{s}\right)+V(\boldsymbol{r}) \Psi\left(\boldsymbol{r}, \boldsymbol{r}_{s}\right)-\frac{\hbar^{2}}{2 \mu_{s}} \nabla_{s}^{2} \Psi\left(\boldsymbol{r}, \boldsymbol{r}_{s}\right) \\
& +V_{s}\left(\boldsymbol{r}_{s}\right) \Psi\left(\boldsymbol{r}, \boldsymbol{r}_{s}\right)=E \Psi\left(\boldsymbol{r}, \boldsymbol{r}_{s}\right)
\end{aligned}
$$

The quantity $\mu$ can be identified with a reduced mass. However, since we are treating spin angular momentum as a particular case of angular momentum therefore we retain the Planck constant and the quantity $\mu_{s}$ also retains the dimension of mass. We call the quantity $\mu_{s}$ an intrinsic mass and it could be related to the curvature that determines the differential geometric and topological structure of a quantum particle, as in the case of Bohr model, or charge. On the other hand, the quantity $V(\boldsymbol{r})$ can be identified with normal potential, such as Coulomb potential but the quantity $V_{s}\left(\boldsymbol{r}_{s}\right)$ represents an intrinsic potential that depends on physical intrinsic properties associated with the spin angular momentum of a quantum particle. Since the two dynamics are independent, the wave equation given in Equation (137) is separable and the total wavefunction $\Psi\left(\boldsymbol{r}, \boldsymbol{r}_{s}\right)$ can be written as a product of two wavefunctions as $\Psi\left(\boldsymbol{r}, \boldsymbol{r}_{s}\right)=\psi(\boldsymbol{r}) \chi\left(\boldsymbol{r}_{s}\right)$. Then Equation (137) is separated into two equations as follows:

$$
\begin{gathered}
-\frac{\hbar^{2}}{2 \mu} \nabla^{2} \psi(\boldsymbol{r})+V(\boldsymbol{r}) \psi(\boldsymbol{r})=E_{0} \psi(\boldsymbol{r}) \\
-\frac{\hbar^{2}}{2 \mu_{s}} \nabla_{s}^{2} \chi\left(\boldsymbol{r}_{s}\right)+V_{s}\left(\boldsymbol{r}_{s}\right) \chi\left(\boldsymbol{r}_{s}\right)=E_{1} \chi\left(\boldsymbol{r}_{s}\right)
\end{gathered}
$$

where $E_{0}+E_{1}=E$. For the case of the hydrogen atom then the total energy spectrum can be found as the sum of two energy spectra as:

$$
E\left(n, n_{s}, m_{s}\right)=-\frac{\mu}{2 \hbar^{2}}\left(\frac{Z q^{2}}{4 \pi \varepsilon_{0}}\right)^{2} \frac{1}{n^{2}}-\frac{A_{s} \mu_{s}}{2 \hbar^{2}\left(n_{s}+m_{s}+\frac{1}{2}\right)^{2}}
$$

It is seen that the total energy spectrum has a fine structure depending on the 
intrinsic quantum numbers $n_{s}$ and $m_{s}$. Furthermore, the total energy spectrum also depends on the undetermined physical quantities $\mu_{s}$ and $A_{s}$ that define the intrinsic properties of a quantum particle, which is the electron in this case. Without restriction, the quantity $\mu_{s}$ can take zero, positive or negative values. Similarly, it is also possible to explain the wave-particle duality by writing simultaneous equations for a quantum particle in the parabolic and hyperbolic relativistic space respectively. If an experiment is designed to detect an invariance associated with a quantum particle which is invariant in the parabolic relativity then it appears as a particle, but if it is invariant in the hyperbolic relativity then it appears as a wave. The formulation of dual properties of particle and wave in two coexisting relativistic spaces may be viewed as a representation of the hidden variable theory and de Broglie theory of double solution in wave mechanics [32] [33].

\section{Conclusion}

We have shown in this work the possibility to classify relativity in accordance with the classification of second order partial differential equations that have been applied into the formulation of physical laws in physics. Based on the classification of second order partial differential equations into hyperbolic, elliptic or parabolic type, we suggested that relativity should also be classified accordingly into hyperbolic, elliptic or parabolic type by establishing coordinate transformations that preserve the forms of the second order partial differential equations. The coordinate transformation that preserves the form of the hyperbolic equation is the Lorentz transformation and the associated space is the hyperbolic, or pseudo-Euclidean, relativistic spacetime. The coordinate transformation that preserves the form of the elliptic equation is the modified Lorentz transformation, or rotation in spacetime, that we have formulated in our work on Euclidean relativity and the associated space is the elliptic, or Euclidean, relativistic spacetime. And the coordinate transformation that preserves the form of the parabolic equation is the Euclidean transformation consisting of the translation and rotation in the spatial space and the associated space is the parabolic relativistic spacetime, which is a Euclidean space with a universal time. Besides the typical equations in physics that comply with hyperbolic relativity such as Maxwell and Dirac equations, we have also established equations that comply with elliptic relativity and these equations can be used to describe the subfields of Maxwell and Dirac field. On the other hand, apart from the typical equations in physics that comply with parabolic relativity such as the diffusion equation, the Schrödinger equation, we have shown that the diffusion equations that are derived from the four-current defined in terms of the differentiable structures of the spacetime manifold and the Ricci flow also belong to parabolic relativity.

\section{Acknowledgements}

We would like to thank the reviewers for their constructive comments and we 
would also like to thank Jane Gao of the administration of JMP for her editorial advice during the preparation of this work.

\section{Conflicts of Interest}

The author declares no conflicts of interest regarding the publication of this paper.

\section{References}

[1] Einstein, A. (1952) The Principle of Relativity. Dover Publications, New York.

[2] Ho, V.B. (2017) Euclidean Relativity.

[3] Schliewe, J. (2019) Open Physics, 17, 731-742. https://doi.org/10.1515/phys-2019-0077

[4] Strauss, W.A. (1992) Partial Differential Equation. John Wiley \& Sons, Inc., New York.

[5] Ho, V.B. (2019) On the Nature of Maxwell and Dirac Field.

[6] Jackson, J.D. (1975) Classical Electrodynamics. John Wiley \& Sons, New York.

[7] Dirac, P.A.M. (1928) Proceedings of the Royal Society A: Mathematical, Physical and Engineering Sciences, 117, 610-624. https://doi.org/10.1098/rspa.1928.0023

[8] Ho, V.B. (2018) International Journal of Physics, 6, 105-115. https://doi.org/10.12691/ijp-6-4-2

[9] Ho, V.B. (2018) GJSFR-A, 18, 37-58.

[10] Ho, V.B. (2018) Journal of Modern Physics, 9, 2402-2419. https://doi.org/10.4236/jmp.2018.914154

[11] Ho, V.B. (2018) Fluid State of the Electromagnetic Field.

[12] Ho, V.B. (2017) On the Motion of Quantum Particles and Euclidean Relativity.

[13] Einstein, A., Podolsky, B. and Rosen, N. (1935) Physical Review, 47, 777-780. https://doi.org/10.1103/PhysRev.47.777

[14] Bell, J.S. (1964) Physics, 1, 195-290. https://doi.org/10.1103/PhysicsPhysiqueFizika.1.195

[15] Rosenfeld, W., Burchardt, D., Garthoff, R., Redeker, K., Ortegel, N., Rau, M. and Weinfurter, H. (2017) Physical Review Letters, 119, Article ID: 010402. https://doi.org/10.1103/PhysRevLett.119.010402

[16] Melshko, S.V. (2005) Methods for Constructing Exact Solutions of Partial Differential Equations. Springer Science \& Business Media, Berlin.

[17] Sobolev, S.L. (1964) Partial Differential Equations of Mathematical Physics. Dover Publications, Inc., New York.

[18] Landau, L.D. and Lifshitz, E.M. (1987) The Classical Theory of Fields. Pergamon Press, Sydney.

[19] Lopes, J.L. (2011) Gauge Field Theories: An Introduction. Pergamon Press, Sydney.

[20] Lim Zheng Liang (2019) Aspects of Newton Cartan Gauge Theory. National University of Singapore, Singapore.

[21] Ho, V.B. (2018) International Journal of Physics, 6, 47-52. https://doi.org/10.12691/ijp-6-2-4

[22] Ho, V.B. (2018) On the Geometric Structure of the Spatiotemporal Manifold.

[23] Ho, V.B. (2019) Maxwell and Dirac Field with Three-Dimensional Time. 
[24] Ho, V.B. (2017) A Derivation of the Ricci Flow.

[25] Ho, V.B. (2017) A Covariant Ricci Flow.

[26] Richard, S. and Hamilton, R.S. (1982) Journal of Differential Geometry, 17, 255-306. https://doi.org/10.4310/jdg/1214436922

[27] Cao, H.-D. and Zhu, X.-P. (2006) The Asian Journal of Mathematics, 10, 165-492. https://doi.org/10.4310/AJM.2006.v10.n2.a2

[28] Ibragimov, N.H., Meleshko, S.V. and Thailert, E. (2008) Communications in Nonlinear Science and Numerical Simulation, 13, 277-284. https://doi.org/10.1016/j.cnsns.2006.03.017

[29] Ibragimov, N.H. (2009) Communications in Nonlinear Science and Numerical Simulation, 14, 1157-1168. https://doi.org/10.1016/j.cnsns.2008.04.010

[30] Ibragimov, N.H. and Meleshko, S.V. (2009) Communications in Nonlinear Science and Numerical Simulation, 14, 2551-2558. https://doi.org/10.1016/j.cnsns.2008.10.007

[31] Ho, V.B. (2019) Journal of Modern Physics, 10, 1374-1393. https://doi.org/10.4236/jmp.2019.1011091

[32] de Broglie, L. (1925) Annales de Physique, 10, 22-128. https://doi.org/10.1051/anphys/192510030022

[33] Bohm, D. (1952) Physical Review, 85, 180-193. https://doi.org/10.1103/PhysRev.85.180 